\title{
Synchronization of tunable asymmetric square-wave pulses in delay-coupled optoelectronic oscillators
}

\author{
Jade Martínez-Llinàs, ${ }^{1}$ Pere Colet, ${ }^{1}$ and Thomas Erneux ${ }^{2}$ \\ ${ }^{1}$ IFISC, Instituto de Física Interdisciplinar y Sistemas Complejos (CSIC-UIB), Campus Universitat Illes Balears, \\ E-07071 Palma de Mallorca, Spain \\ ${ }^{2}$ Université Libre de Bruxelles, Optique Nonlinéaire Théorique, Campus Plaine, C.P. 231, 1050 Bruxelles, Belgium
}

(Received 13 October 2014; published 18 March 2015)

\begin{abstract}
We consider a model for two delay-coupled optoelectronic oscillators under positive delayed feedback as prototypical to study the conditions for synchronization of asymmetric square-wave oscillations, for which the duty cycle is not half of the period. We show that the scenario arising for positive feedback is much richer than with negative feedback. First, it allows for the coexistence of multiple in- and out-of-phase asymmetric periodic square waves for the same parameter values. Second, it is tunable: The period of all the square-wave periodic pulses can be tuned with the ratio of the delays, and the duty cycle of the asymmetric square waves can be changed with the offset phase while the total period remains constant. Finally, in addition to the multiple inand out-of-phase periodic square waves, low-frequency periodic asymmetric solutions oscillating in phase may coexist for the same values of the parameters. Our analytical results are in agreement with numerical simulations and bifurcation diagrams obtained by using continuation techniques.
\end{abstract}

DOI: 10.1103/PhysRevE.91.032911

PACS number(s): 05.45.Xt, 42.65.Sf, 85.60.-q

\section{INTRODUCTION}

Dynamical systems with delay are ubiquitous in nature, and they have been the topic of research in science and technology during the past few decades. For large delay, namely when the delay is much larger than the other time scales of the system, square-wave periodic solutions emerge as the dominant solutions for specific values of the parameters [1]. Square-wave switching in optical and optoelectronic systems has been studied during the past few years [2-10], not only for its fundamental interest but also motivated by applications such as optical clocks [2] or optical sensing [3]. In particular, stable square-wave switching has been observed in edge-emitting diode lasers (EELs) [4-6], vertical cavity surface-emitting lasers (VCSELs) $[7,8,11]$, optoelectronic oscillators (OEOs) $[12,13]$, ring lasers [9], and mode-locked fiber lasers [10,14].

Square-wave pulses with asymmetric duty cycle and a period close to but longer than twice the delay time has been obtained in mutually coupled EELs subject to polarizationrotated feedback (PROF) [5,6], in ring lasers [9], and in a single OEO [13]. The square waves generated with mutually EELs under PROF were always in antiphase. In this work we study a system of two mutually coupled OEOs for asymmetric squarewave pulse generation. OEOs can be designed to be very robust to noise and exhibit periodic microwaves with exceptional purity [15-20] or to generate high-bandwith chaotic output $[21,22]$. An advantage of using mutually coupled OEOs with respect to mutually coupled EELs is that OEOs can produce both synchronized in-phase and out-of phase square waves. Furthermore, we will show that two mutually coupled OEOs can generate asymmetric square-wave switching even when they are identically coupled, in contrast with ring lasers which need to have asymmetric couplings to produce asymmetric square waves.

The generation of periodic square waves of controllable period is an interesting problem which was already addressed in the past using the Ikeda paradigm [23-25], which presents a nonlinear feedback proportional to $\cos ^{2}\left(x_{D}+\Phi\right)$, where $x_{D}$ is a delayed variable and $\Phi \in[-\pi / 2, \pi / 2]$ is a constant offset phase which fixes the operating point. For $\Phi \in[0, \pi / 2]$ the feedback term tends to reduce small disturbances, and thus the feedback can be qualified as negative. In this situation square waves are symmetric in the sense that the duty cycle is half the period. For $\Phi \in[-\pi / 2,0]$ the feedback term tends to increase small disturbances, namely the feedback is positive. In this situation square waves are in general asymmetric in the sense that the duty cycle differs from half the period.

We have recently studied the generation of symmetric square-wave pulses in a model for two mutually delay-coupled OEOs with offset phases in the range for negative feedback [26]. We showed that multiple in-phase square waves with different periods can coexist when the ratio between the self-feedback and the cross-feedback delay times satisfies a rational relationship involving two odd numbers. Similarly, multiple out-of-phase square waves with different periodicity can coexist when the ratio between the self- and the cross-delay times is an odd-even rational. It was also found that the symmetric square-wave periodic solutions undergo a period doubling route to chaos.

In this paper we study the emergence of stable asymmetric square waves in the same model but in the case where the offset phases are in the range for positive feedback. The asymmetric square waves arising with positive feedback turn out to be more flexible and robust than with negative feedback; in particular, their asymmetry allows them to adapt their shape in response to offset phase changes instead of undergoing a period doubling. We find that the system has stable in- and out-of-phase synchronized solutions and that several solutions can coexist similarly to the case of negative feedback. Nevertheless, in contrast to the negative feedback case, here out-of-phase solutions always coexist with in-phase solutions with different periods. Furthermore, we show that the duty cycle of the square waves generated with positive feedback can be tuned with the offset phase, while the total period remains constant. Finally, there are low-frequency limit 
cycles, microwave oscillations, synchronized in-phase and coexisting with the above-mentioned fast square waves for the same values of the parameters.

For synchronization of systems with multiple delays, the ratio between different delay times plays a crucial role, as it has recently been shown for mutually coupled chaotic systems [27]. Here we focus on the tunability of multiple stable periodic solutions generated by the mutually coupled OEOs rather than their synchronization efficiency in the chaotic regime. We use asymptotic methods based on the large values of the delay times compared to the other time scales of the system to analyze their bifurcation mechanisms. We show that the period of the square-wave pulses is determined by the ratio between the two delays. Our analytical results are substantiated with numerical simulations of the full dynamical model.

The outline of the article is the following. The model is presented in Sec. II. In Sec. III we study the Hopf bifurcations of the steady state, in Sec. IV we show some numerical simulations of periodic square waves with positive feedback, in Sec. V we obtain an analytic approximation for the periodic square-wave solutions, in Sec. VI we compare the analytical results with numerical simulations, in Sec. VII we study the effect of a small mismatch in the delay times, in Sec. VIII we analyze the effect of different self- and cross-feedback strengths, and the last section concludes the paper.

\section{MODEL}

We model two OEOs that are mutually coupled as schematically shown in Fig. 1. The light emitted by a cw semiconductor laser (LD) with intensity $P$ is split into two equal parts, each part feeding an electro-optical delay loop. The optical part of the loop consists of a Mach-Zehnder interferometer (MZI) and an optical delay line, whereas the electronic circuit is

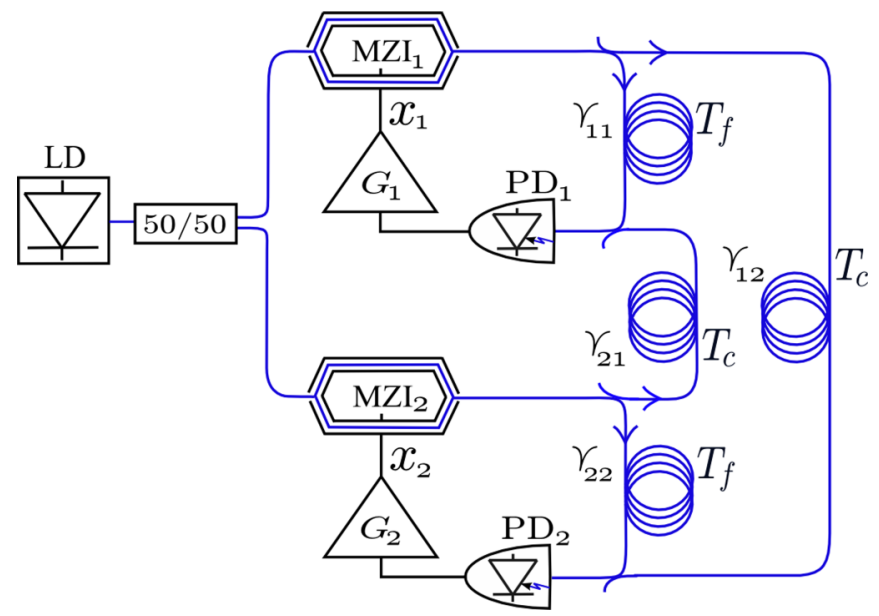

FIG. 1. (Color online) Schematic diagram of the system we are modeling consisting of two mutually coupled OEOs. Each OEO consists of a Mach-Zehnder interferometer labeled as MZI, a fiber delay loop with a delay time $T_{f}$, a photodiode labeled as PD, and a RF amplifier labeled as $G$ whose output modulates one of the arms of the Mach-Zehnder. The OEOs are fed by a laser diode, labeled as LD, whose output is split in two parts by a 50-50 fiber splitter. The two OEO are mutually coupled with a cross-feedback delay time $T_{c}$. composed by a photodiode (PD) and an amplifier [21,22]. We use subindex $i, i=1,2$, to identify the variables associated to loop $i$. For loop $i$ the optical output of MZI $_{i}$ is split into two parts. A fraction $\alpha_{i i}$ is delayed using a fiber loop by a time $T_{i i}$. A fraction $\alpha_{i j}$ with $i, j=1,2$ and $j \neq i$ is injected from loop $i$ into loop $j$ after a delay $T_{i j}$. Self-feedback and cross-feedback optical signals are combined and the resulting intensity is detected by the PD. The electrical signal is band-pass amplified and used to drive the Mach-Zehnder AC electrode. For each loop, the dynamics results from a combination of the nonlinear effect due to the MZI plus a linear filtering process associated to the electrical circuit $[21,22]$.

The dynamics can be described in terms of $x_{i}$, proportional to the ac voltage applied to the MZI, and $y_{i}(t)=\int_{t_{0}}^{t} x_{i}\left(t^{\prime}\right) d t^{\prime}$, leading to a system of four delay differential equations [26],

$$
\begin{aligned}
\tau_{i} \dot{x}_{i}(t)= & -x_{i}(t)-\theta_{i}^{-1} y_{i}(t) \\
& +P\left\{\gamma_{i i}^{2} \cos ^{2}\left[x_{i}\left(t-T_{i i}\right)+\Phi_{i}\right]\right. \\
& +\gamma_{j i}^{2} \cos ^{2}\left[x_{j}\left(t-T_{j i}\right)+\Phi_{j}\right] \\
& +2 \gamma_{i i} \gamma_{j i} \cos \left[x_{j}\left(t-T_{j i}\right)+\Phi_{j}\right] \\
& \times \cos \left[x_{i}\left(t-T_{i i}\right)+\Phi_{i}\right] \cos \left[x_{i}\left(t-T_{i i}\right)+\Phi_{i}\right. \\
& \left.\left.-x_{j}\left(t-T_{j i}\right)-\Phi_{j}+(-1)^{i} \Phi_{0}\right]\right\} \\
\dot{y}_{i}(t)= & x_{i}(t),
\end{aligned}
$$

where $i, j=1,2, j \neq i, \theta_{i}$, and $\tau_{i}$ are the band-pass filter characteristic times, $\Phi_{i}$ is an offset phase proportional to the $\mathrm{dc}$ voltage applied to $\mathrm{MZI}_{i}, \Phi_{0}$ is the phase difference between the nonmodulated arms of the two $\mathrm{MZI}_{s}$, and $\gamma_{i j}$ are effective feedback strengths.

For simplicity, in this work we consider the case in which the two systems have identical parameters: $\theta_{1}=\theta_{2}=\theta, \tau_{1}=$ $\tau_{2}=\tau, \Phi_{1}=\Phi_{2}=\Phi, T_{11}=T_{22}=T_{f}$. We also consider $\Phi_{0}=0$ and define the coupling time as $T_{c}=\left(T_{21}+T_{12}\right) / 2$. The steady-state solution is given by

$$
x_{i_{\mathrm{st}}}=0, \quad y_{i_{\mathrm{st}}}=\theta P\left(\gamma_{i i}+\gamma_{j i}\right)^{2} \cos ^{2} \Phi .
$$

As in Ref. [26], introducing $Y_{i}(t)=\left[y_{i}(t)-y_{i_{\mathrm{st}}}\right] / T_{c}$ and $s=$ $t / T_{c}$,

$$
\begin{aligned}
\varepsilon x_{i}^{\prime}(s)= & -x_{i}(s)-\delta Y_{i}(s)+\frac{P}{2}\left\{-\left(\gamma_{i i}+\gamma_{j i}\right)^{2} \cos (2 \Phi)\right. \\
& +\left(\gamma_{i i}+\gamma_{j i}\right)\left\{\gamma_{i i} \cos \left[2 x_{i}\left(s-s_{0}\right)+2 \Phi\right]\right. \\
& \left.+\gamma_{j i} \cos \left[2 x_{j}(s-1)+2 \Phi\right]\right\} \\
& \left.+\gamma_{j i} \gamma_{i i} \cos \left[2 x_{i}\left(s-s_{0}\right)-2 x_{j}(s-1)\right]\right\} \\
Y_{i}^{\prime}(s)= & x_{i}(s)
\end{aligned}
$$

where prime means differentiation with respect to $s$ and

$$
s_{0}=T_{f} / T_{c}, \quad \varepsilon=\tau / T_{c}, \quad \delta=T_{c} / \theta .
$$

For most of this work, we will also consider that the self- and cross-feedback strength coefficients are the same. The case of different feedback strength coefficients will be discussed in 
Sec. VIII. Taking $\gamma_{11}=\gamma_{22}=\gamma_{12}=\gamma_{21}=\gamma$, Eqs. (3) read

$$
\begin{aligned}
\varepsilon x_{i}^{\prime}(s)= & -x_{i}(s)-\delta Y_{i}(s)+P \gamma^{2}\left\{\cos \left[2 x_{i}\left(s-s_{0}\right)+2 \Phi\right]\right. \\
& +\cos \left[2 x_{j}(s-1)+2 \Phi\right]-2 \cos (2 \Phi) \\
& \left.+\cos \left[2 x_{i}\left(s-s_{0}\right)-2 x_{j}(s-1)\right]\right\} \\
Y_{i}^{\prime}(s)= & x_{i}(s) .
\end{aligned}
$$

We note that Eqs. (5) are invariant to the symmetry $x_{i} \rightarrow$ $-x_{i}, \Phi_{i} \rightarrow-\Phi_{i}-\frac{\pi}{2}$, provided that

$$
x_{j}\left(s-s_{0}\right)=x_{i}(s-1),
$$

where $i, j=1,2, j \neq i$. As discussed in Sec. $\mathrm{V}$, this condition is fulfilled by solutions exhibiting generalized lag synchronization.

Equations (5) admit time-periodic square-wave solutions which are born as sinusoidal periodic solutions from a Hopf bifurcation of the steady state. In the next two sections we analyze these Hopf bifurcations and the emerging periodic solutions. We consider that the delay time $T_{c}$ has a value of the order of tens of nanoseconds and that the band-pass filter has characteristic times $\theta=5 \mu$ s and $\tau=25$ ps. Therefore $\varepsilon$ is of order $10^{-4}$ and $\delta$ of order $10^{-2}$.

\section{ONSET OF PERIODIC SOLUTIONS}

Proceeding in a similar way as in Ref. [26], in this section we determine the Hopf bifurcations of the zero solution. We consider $x_{i}(s)=x_{i}^{\text {st }}+u_{i}(s)$ and $Y_{i}(s)=Y_{i}^{\text {st }}+v_{i}(s)$ and formulate the linearized equations for the small perturbations $u_{i}$ and $v_{i}$,

$$
\begin{aligned}
\varepsilon u_{i}^{\prime}(s) & =-u_{i}(s)-\frac{\chi}{2}\left[u_{i}\left(s-s_{0}\right)+u_{j}(s-1)\right]-\delta v_{i} \\
v_{i}^{\prime}(s) & =u_{i}(s),
\end{aligned}
$$

where

$$
\chi \equiv 4 P \gamma^{2} \sin (2 \Phi)
$$

is an effective bifurcation parameter as seen below. $\chi$ is negative for positive feedback $(\Phi \in[-\pi / 2,0])$. We note that in the stability analysis done in Ref. [26] $\delta$ was neglected because it was a small parameter. However, here we keep it because it will be necessary to determine the frequency of a slow oscillation as we will see below. We look for solutions of (7) of the form $u_{i}=c_{i} \exp [(\lambda+i \omega) s], v_{i}=d_{i} \exp [(\lambda+i \omega) s]$. At the Hopf bifurcation $\lambda=0$, and the linearized problem reduces to a homogeneous system of two linear algebraic equations for $c_{1}$ and $c_{2}$ :

$$
\begin{aligned}
& 0=c_{1}\left(1+i \omega \varepsilon-i \frac{\delta}{\omega}+\frac{\chi}{2} e^{-i \omega s_{0}}\right)+c_{2} \frac{\chi}{2} e^{-i \omega} \\
& 0=c_{1} \frac{\chi}{2} e^{-i \omega}+c_{2}\left(1+i \omega \varepsilon-i \frac{\delta}{\omega}+\frac{\chi}{2} e^{-i \omega s_{0}}\right) .
\end{aligned}
$$

The condition of a nontrivial solution gives rise to the characteristic equation

$$
1+i \omega \varepsilon-i \frac{\delta}{\omega}+\frac{\chi}{2}\left(e^{-i \omega s_{0}} \pm e^{-i \omega}\right)=0 .
$$

Replacing (10) into (9) we obtain $c_{1}= \pm c_{2}$. Therefore there are two different families of Hopf bifurcations. The + sign leads to in-phase oscillations of $x_{1}$ and $x_{2}$ while for the - sign oscillations are out of phase.

Splitting (10) into real and imaginary part gives rise two equations for $\chi$ and $\omega$ :

$$
\begin{aligned}
1+\frac{\chi}{2}\left[\cos \left(\omega s_{0}\right) \pm \cos (\omega)\right] & =0, \\
\omega \varepsilon-\frac{\delta}{\omega}-\frac{\chi}{2}\left[\sin \left(\omega s_{0}\right) \pm \sin (\omega)\right] & =0 .
\end{aligned}
$$

For in-phase oscillations (11) and (12) can be written as

$$
\begin{aligned}
& \chi \cos \left(\omega \frac{s_{0}+1}{2}\right) \cos \left(\omega \frac{s_{0}-1}{2}\right)=-1, \\
& \chi \sin \left(\omega \frac{s_{0}+1}{2}\right) \cos \left(\omega \frac{s_{0}-1}{2}\right)=\omega \varepsilon-\frac{\delta}{\omega} .
\end{aligned}
$$

Dividing (14) by (13) leads to

$$
\tan \left(\omega \frac{s_{0}+1}{2}\right)=\frac{\delta}{\omega}-\omega \varepsilon
$$

Since $\delta$ and $\varepsilon$ are small, the argument of the tangent must be close to a multiple of $\pi$. Therefore the frequency of the in-phase oscillations is given by

$$
\omega_{n}^{\text {in }}=\frac{2 n \pi}{s_{0}^{\text {in }}+1}+\alpha_{n}^{\text {in }}(\varepsilon, \delta),
$$

where $\alpha_{n}^{\text {in }}$ is a correction of order $O(\varepsilon, \delta)$. For $n \geqslant 1, \alpha_{n}^{\text {in }}$ can be neglected since it is much smaller than $\frac{2 n \pi}{s_{0}^{\mathrm{n}}+1}$. Thus to good approximation we can take

$$
\omega_{n}^{\text {in }}=\frac{2 n \pi}{s_{0}^{\text {in }}+1}, \quad n \geqslant 1 .
$$

For the case $n=0$, replacing (16) in (15) and expanding the tangent we get

$$
\omega_{0}^{\text {in }}=\sqrt{\frac{2 \delta}{s_{0}+1+2 \varepsilon}} \approx \sqrt{\frac{2 \delta}{s_{0}+1}} .
$$

These are oscillations at a low frequency which is determined mainly by $\delta$ and, for typical parameter values, is in the microwave regime. These slow oscillations arising from a Hopf instability of the zero solution are also found in a single OEO with positive feedback $[12,28]$ and can be used for pure microwave generation $[15,16,19,20]$. The period of the microwave oscillations can be much larger than the delay times present in the system and therefore they do not take the shape of square waves, as illustrated in Sec. IV. For this reason here we will be mainly interested in the case $n \geqslant 1$.

Similarly, for out-of phase oscillations (11) and (12) can be written as

$$
\begin{aligned}
& \chi \sin \left(\omega \frac{s_{0}+1}{2}\right) \sin \left(\omega \frac{s_{0}-1}{2}\right)=1, \\
& \chi \cos \left(\omega \frac{s_{0}+1}{2}\right) \sin \left(\omega \frac{s_{0}-1}{2}\right)=\omega \varepsilon-\frac{\delta}{\omega} .
\end{aligned}
$$

Dividing (19) by (20) leads to

$$
\cot \left(\omega \frac{s_{0}+1}{2}\right)=\frac{\delta}{\omega}-\omega \varepsilon .
$$


In the limit of small $\delta$ and $\varepsilon$ the argument of the cotangent must be closer to $n \pi+\pi / 2$. Thus out-of-phase oscillations have a frequency given by

$$
\omega_{n}^{\text {out }}=\frac{(2 n+1) \pi}{s_{0}^{\text {in }}+1}+\alpha_{n}^{\text {out }}(\varepsilon, \delta),
$$

where $\alpha_{n}^{\text {out }}$ is a correction of order $O(\varepsilon, \delta)$ which much smaller than $\frac{(2 n+1) \pi}{s_{0}^{\text {in }}+1}$ for any $n$, including $n=0$. Thus there are no outof-phase microwave oscillations. Disregarding the correction $\alpha_{n}^{\text {out }}$ we will take

$$
\omega_{n}^{\text {out }}=\frac{(1+2 n) \pi}{s_{0}^{\text {out }}+1}, \quad n \geqslant 0 .
$$

We now determine the threshold for the onset of oscillations. For in-phase microwave oscillations, replacing (18) into (13) leads to

$$
\begin{aligned}
\chi_{0}^{\text {in }} & =-\frac{1}{\cos \left[\sqrt{\frac{\delta\left(s_{0}+1\right)}{2}}\right] \cos \left[\left(s_{0}-1\right) \sqrt{\frac{\delta}{2\left(s_{0}+1\right)}}\right]} \\
& \approx-1-\frac{\delta}{2} \frac{s_{0}^{2}+1}{s_{0}+1} .
\end{aligned}
$$

Thus, the Hopf bifurcation associated to microwave oscillations can only take place for positive feedback. As for fast in-phase oscillations, replacing (17) into (13) leads to

$$
\chi_{n}^{\text {in }}=-\frac{1}{\cos \left(\omega_{n}^{\text {in }}\right)}, \quad n \geqslant 1 .
$$

Finally, for out-of-phase oscillations, replacing (23) into (19) and using that $\sin (a+\pi / 2)=\cos (a)$ we obtain

$$
\chi_{n}^{\text {out }}=\frac{1}{\cos \left(\omega_{n}^{\text {out }}\right)}, \quad n \geqslant 0 .
$$

Therefore fast in-phase and out-of phase oscillations can appear both for positive and negative feedback provided $|\chi| \geqslant 1$. The case of negative feedback has been considered in Ref. [26]. Here we focus on the case of positive feedback. Taking $s_{0}$ as a parameter there is a family of Hopf bifurcation curves $\chi_{n}\left(s_{0}\right)$ which are plotted in Fig. 2 for $n=1,2,3$ for the in-phase and out-of-phase solutions. All the curves have a maximum at $\chi_{n}=-1$ and close to the maximum have a parabolic shape. Increasing $n$ the parabolic shape narrows, curves have more maxima, and maxima are located closer.

The Hopf bifurcations appearing at $\chi_{n}=-1$ for a given value of $s_{0}$ are particularly relevant from the physical point of view since these are the first instabilities that appear increasing the LD power in a system with given delay times. Setting $\chi_{n}=-1$ in (25) and (26) we have $\omega_{n}^{\text {in }}=2 m \pi(m=1,2, \ldots)$ and $\omega_{n}^{\text {out }}=(2 m+1) \pi(m=0,1,2 \ldots)$, respectively, for in and out-of-phase fast oscillations. Therefore the period is given by

$$
\begin{aligned}
& T^{\text {in }}=\frac{1}{m}, \\
& T^{\text {out }}=\frac{2}{2 m+1} .
\end{aligned}
$$

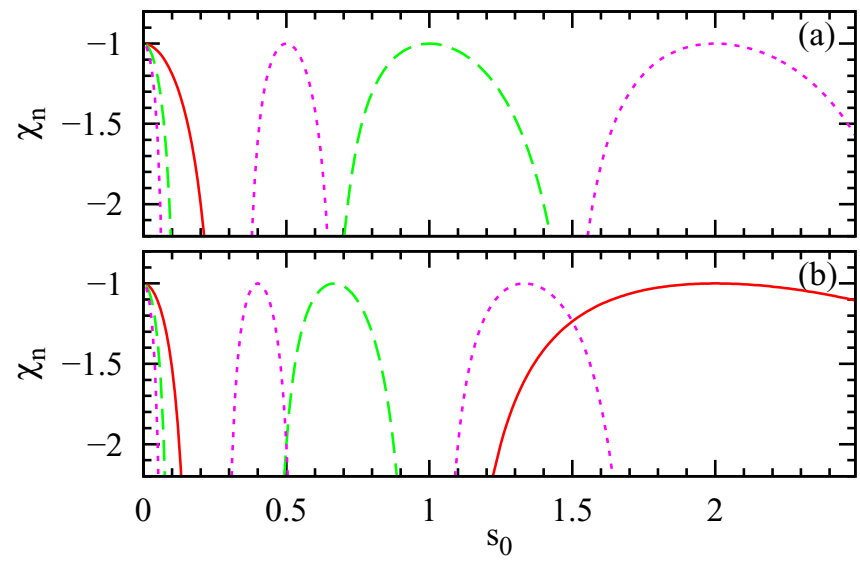

FIG. 2. (Color online) Hopf bifurcation curves with $\chi_{n}<0$ for the in-phase (top panel) and out-of-phase (bottom panel) solutions with $n=1$ (continuous red), $n=2$ (dashed green), and $n=3$ (dotted pink).

From (17) and (23), we then determine the following values of $s_{0}$ :

$$
\begin{aligned}
s_{0}^{\text {in }} & =\frac{n-m}{m}=\frac{k}{m}, \\
s_{0}^{\text {out }} & =\frac{2(n-m)}{2 m+1}=\frac{2 k}{2 m+1},
\end{aligned}
$$

where $k \equiv n-m$. The condition $s_{0}>0$ implies $k \geqslant 1$ and restricts the value of $m$ to the range $0<m<n$ and $0 \leqslant m<n$ for in- and out-of-phase solutions, respectively.

From (27)-(30) we have

$$
\begin{gathered}
s_{0}^{\text {in }}=k T^{\text {in }}, \\
1-s_{0}^{\text {in }}=\frac{m-k}{m}=(m-k) T^{\text {in }}, \\
s_{0}^{\text {out }}=k T^{\text {out }}, \\
1-s_{0}^{\text {out }}=\frac{2(m-k)+1}{2 m+1}=\left(m-k+\frac{1}{2}\right) T^{\text {out }} .
\end{gathered}
$$

Thus, the dimensionless time difference $\left(T_{c}-T_{f}\right) / T_{c}=1-$ $s_{0}$ can be any rational number for in-phase periodic solutions, whereas it has to be an odd-odd rational number for out-ofphase solutions.

A few observations are worth pointing out. First, for all the values of $s_{0}$ satisfying (30) and thus allowing for out-of-phase solutions they do also satisfy (29) and therefore out-of-phase solutions always coexist with in-phase solutions. The coexistence of in- and out-of-phase Hopf bifurcation points for the same $s_{0}$ that appears with negative feedback $\left(\chi_{n}<0\right)$ it is not allowed with positive feedback $\left(\chi_{n}>0\right)$ as it was recently demonstrated in Ref. [26]. Second, several in-phase Hopf bifurcations or several out-of phase solutions may appear for the same value of $s_{0}$ with different periods. This means that the Hopf bifurcation at $\chi_{n}=-1$ can be multiple. These two results are illustrated in Fig. 3, where the period of Hopf bifurcation points emerging from $\chi_{n}=-1$ is plotted as a 


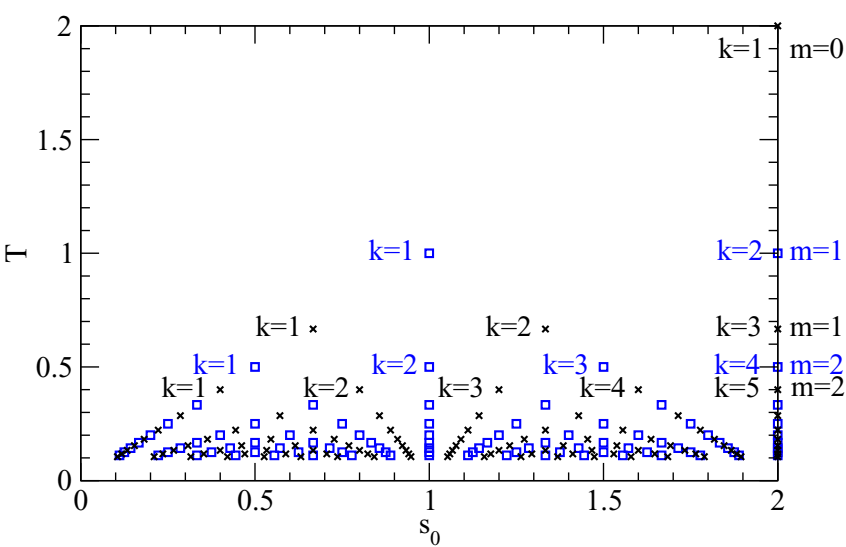

FIG. 3. (Color online) Hopf bifurcation points appearing at $\chi_{n}=$ -1 as a function of $s_{0}$ leading to fast oscillatory solutions with different periods $T$. Squares and crosses correspond to in- and out-of-phase Hopf bifurcations, respectively. As $T$ approaches zero, the number of Hopf bifurcations increases fast (only bifurcations with $T \geqslant 0.1$ are shown)

function of $s_{0}$. In this figure, points with the same $m$ are located in horizontal lines, points with the same $k$ are located in straight lines of slope $1 / k$ starting from the origin $\left(s_{0}=0, T=0\right)$, whereas points with the same $m-k$ are located in straight lines that start at $\left(s_{0}=1, T=0\right)$.

Regarding coexistence of solutions, we note that once $s_{0}$ is fixed only certain values of $k$ and $m$ are possible. For a given $s_{0}$ the fundamental in-phase nanosecond solution is the one that corresponds to the minimum values of $k$ and $m$ that fulfill (29) which we label as $k_{1}$ and $m_{1}$. Higher harmonics are obtained by multiplying numerator and denominator of (29) by any positive integer number. This is in contrast with the system with negative feedback, for which only odd multipliers were allowed [26]. The values of $m$ and $k$ for the in-phase harmonic of order $j-1$ are given by

$$
m_{j}=j m_{1}, k_{j}=j k_{1}, j=1,2, \ldots
$$

and the period by

$$
T_{j}^{\text {in }}=\frac{T_{1}^{\text {in }}}{j}=\frac{s_{0}}{j k_{1}} .
$$

Note that $j=1$ corresponds to the fundamental in-phase nanosecond solution, $j=2$ to the first harmonic and so on.

The out-of-phase fundamental solution corresponds to the minimum values of $k$ and $m$ that fulfill (30) which we label as $k_{0}$ and $m_{0}$. Multiplication of numerator and denominator by an even number leads to a ratio that does not fulfill (30). Therefore, similarly to what was encountered for negative feedback [26], only odd multipliers are allowed. The harmonic of order $j$ is obtained by multiplying numerator and denominator of $(30)$ by $(2 j+1)$. Therefore, the values allowed for $m$ and $k$ are given by

$$
m_{j}=(2 j+1) m_{0}+j, k_{j}=(2 j+1) k_{0}, j=0,1,2, \ldots
$$

The period of harmonic $j$ is

$$
T_{j}^{\text {out }}=\frac{T_{0}^{\text {out }}}{2 j+1}=\frac{s_{0}}{(2 j+1) k_{0}} .
$$

\section{NUMERICAL SIMULATIONS OF PERIODIC SOLUTIONS WITH POSITIVE FEEDBACK}

The solutions of (5) with $\Phi>0$ have already been analyzed in Ref. [26]. Here we complete the study with numerical simulations of the dynamical model (5) for $\Phi<0$. We encounter that the zero solution becomes unstable at $\chi=-1$ for any value of $s_{0}$ leading to multiple fast oscillatory solutions, the fundamental and the harmonics, with a fundamental period of the order of tens of nanoseconds. We first focus on the fundamental in-phase nanosecond solution $\omega_{1}^{\text {in }}$. Just after the bifurcation at $\chi=-1$ the nanosecond periodic solution is sinusoidal, as shown in Fig. 4(a) for $\chi=-1.0001$. The sinusoidal shape is soon degraded [see Fig. 4(b) for $\chi=1.001$ ] and already for $\chi=1.01$ [Fig. 4(c)] the solution is almost a square wave with two tilted plateaus and sharp transition layers; increasing $\chi$ further the transition layers become sharper and the plateaus become practically horizontal as shown in Fig. 4(d) for $\chi=1.1$. In Fig. 4 we have only plotted $x_{1}$ for clarity; for the values of the delays used in Fig. 4, $x_{2}$ is synchronized in phase with $x_{1}$. For $\Phi=-0.25 \pi$ the nanosecond oscillatory solution appears supercritically. For
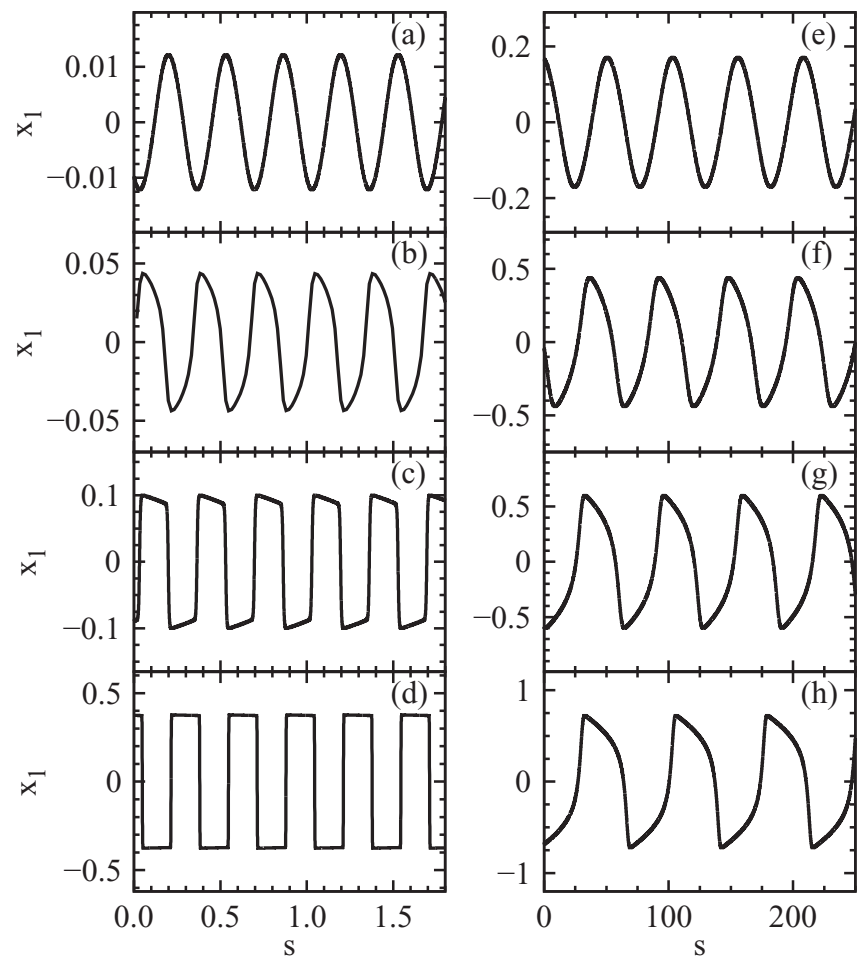

FIG. 4. Periodic solutions with $\gamma=0.5, \Phi=-0.25 \pi, T_{f}=$ $40 \mathrm{~ns}$, and $T_{c}=60 \mathrm{~ns}$, so $s_{0}=2 / 3, \varepsilon=4.17 \times 10^{-4}$, and $\delta=0.012$. The panels on the left depict nanosecond periodic solutions with $\chi=-1.0001$ (a), $\chi=-1.001$ (b), $\chi=-1.01$ (c), and $\chi=-1.1$ (d). The panels on the right depict microsecond periodic solutions with $\chi=-1.0104$ (e), $\chi=-1.03$ (f), $\chi=-1.106(\mathrm{~g})$, and $\chi=$ $-1.3(\mathrm{~h})$. 


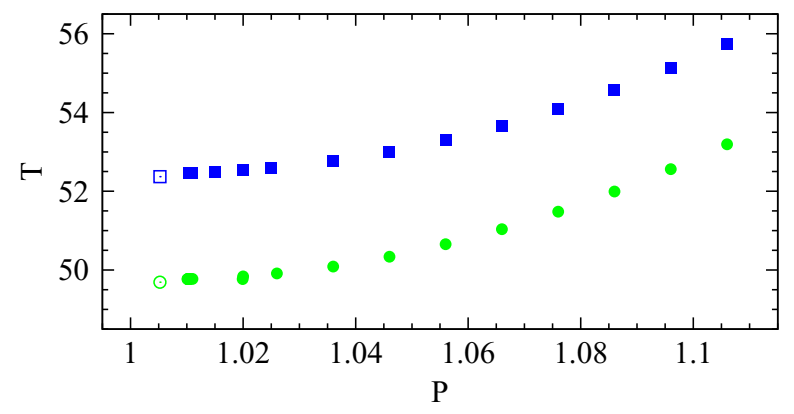

FIG. 5. (Color online) Period of microwaves with $\gamma=0.5$ and $\Phi=-0.25 \pi$ as a function of the laser power. Blue filled squares and green filled circles correspond to numerical simulations with $s_{0}=2 / 3\left(T_{f}=40 \mathrm{~ns}\right.$ and $\left.T_{c}=60 \mathrm{~ns}\right)$ and $s_{0}=1 / 2\left(T_{f}=30 \mathrm{~ns}\right.$ and $\left.T_{c}=60 \mathrm{~ns}\right)$, respectively. Empty symbols depict the Hopf bifurcation point to microwaves $\chi_{0}^{\text {in }}\left(s_{0}\right)$.

other values of the offset phase the solutions can appear subcritically.

Another bifurcation takes place at $\chi_{0}^{\text {in }}\left(s_{0}\right)$ towards in-phase periodic solutions with period of the order of microseconds, corresponding to $\omega_{0}^{\text {in }}$. Microwaves are born unstable, as discussed below; however, by increasing $|\chi|$ they soon become stable. Figure 4(e) shows the stable microwaves found for $\chi=-1.0104$. For values of $\chi$ close to -1 , microwaves have a sinusoidal shape. By increasing $|\chi|$ the sinusoidal shape is degraded, as can be seen in Figs. 4(f), 4(g), and 4(h) with $\chi=-1.03, \chi=-1.106$, and $\chi=-1.3$, respectively. The shape of the microsecond solutions shown in Fig. 4 is not square wave because the period of the solutions is large compared to the delay times present in the system. As reflected in Figs. 4(e) and 4(f) the period of the microwaves shows a strong dependence with $\chi$ contrary to the fast oscillations for which the period remains practically constant as $|\chi|$ increases. More quantitatively, Fig. 5 shows how the period of microwaves increases with the pump close to $\chi=-1$. The empty symbols correspond to the Hopf instability $\chi_{0}^{\text {in }}\left(s_{0}\right)$. The filled symbols correspond to stable microwaves obtained from numerical simulations. The prediction of the period close to the Hopf is excellent. Further away the period continues to increase with a nonlinear dependence with $P$.

As stated before, microwave solutions are born unstable, thus approaching $\chi_{0}^{\text {in }}\left(s_{0}\right)$ from above they become unstable slightly before reaching the threshold value. For delay times whose ratio allows for out-of-phase oscillations, microwaves acquire an out-of-phase modulation with period $T_{0}^{\text {out }}$. For instance, for $T_{f}=40 \mathrm{~ns}$ and $T_{c}=60 \mathrm{~ns}\left(s_{0}=2 / 3\right)$, the microwave becomes unstable at $\chi=-1.01036$, before reaching the threshold $\chi_{0}^{\text {in }}=-1.0052$, as shown in Fig. 6. Figures 6(a), $6(\mathrm{~b}), 6(\mathrm{c})$, and $6(\mathrm{~d})$ show the onset of the instability. This is a long transient, obtained after time $2 \times 10^{5} T_{c}$. The final state is the fundamental out-of-phase square wave shown in Figs. 6(e) and 6(f). For delay times whose ratio allows only for in-phase oscillations, such as $T_{f}=30 \mathrm{~ns}$ and $T_{c}=60 \mathrm{~ns}$ $\left(s_{0}=1 / 2\right)$, the microwave solution has a slightly larger range of stability, up to $\chi=-1.00992$. Nevertheless, it finally destabilizes before reaching the threshold value $\chi_{0}^{\text {in }}=-1.005$. In this case the instability originates as an in-phase modulation

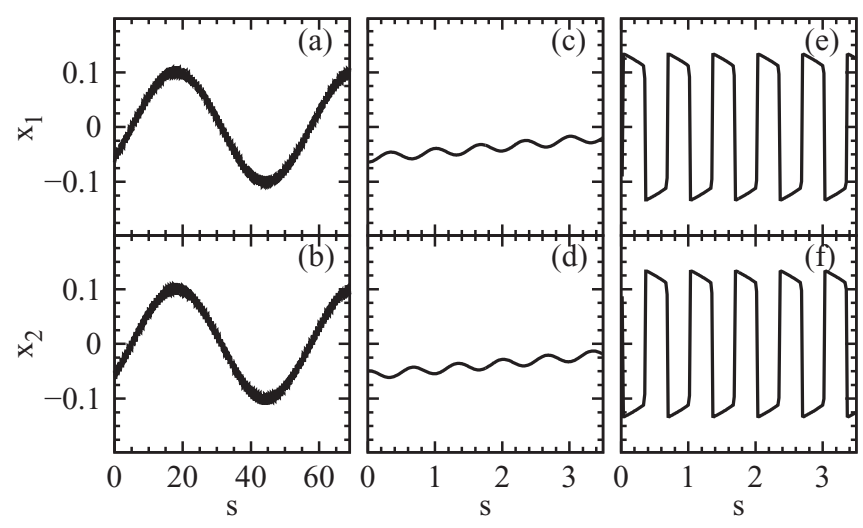

FIG. 6. Numerical solutions with $P=1.01036, \gamma=0.5, \Phi=$ $-0.25 \pi, T_{f}=40 \mathrm{~ns}$, and $T_{c}=60 \mathrm{~ns}$, so $s_{0}=2 / 3, \varepsilon=4.17 \times 10^{-4}$, and $\delta=0.012$ starting from a microwave oscillation as initial condition. Panels (a) and (b), with a zoom in (c) and (d), show the onset of the fast out-of-phase instability. Panels (e) and (f) show the final nanosecond periodic solutions after a long transient.

with period $T_{1}^{\text {in }}$ and leads to the fundamental in-phase square wave.

Figure 7 shows the microwave periodic solutions obtained with different values of $\Phi<0$ for a larger pump ( $P=1.5)$. In this figure $x_{1}$ and $x_{2}$ are plotted in black and pink dots, respectively. The zooms plotted in the right column show that microwaves are always perfectly in phase, as predicted theoretically. Microwaves are symmetric only for $\Phi=-\pi / 4$. The period shows a strong dependence with the offset phase, being minimum for the symmetric solution [Fig. 7(e)] and increasing while the the shape becomes more and more asymmetric as $\Phi$ is moved away from $\Phi=-\pi / 4$. This is discussed in more detail in Sec. VI. Notice that since in-phase microwaves satisfy (6) there is a symmetry $x_{i} \rightarrow-x_{i}$, $\Phi_{i} \rightarrow-\Phi_{i}-\frac{\pi}{2}$, in the time traces shown in Fig. 7 .

We now focus on the fast square-wave solutions. For an even-odd ratio between self- and cross-delay, in- and out-of-phase square waves coexist, as predicted in Sec. III. To illustrate this situation, Figs. 8 and 9 show, respectively, the in-phase and the out-of-phase fundamental square waves, both coexisting for $s_{0}=2 / 3, P=1.5, \gamma=0.5$, and different offset phases $\Phi$. As it can be seen from these figures, the periodic square waves are symmetric for $\Phi=-\pi / 4$; namely they take a plateau value $-x^{*}$ for half of the period and a value $x^{*}$ for the other half. For other offset phases the shape is asymmetric with a duty cycle which depends on the offset phase. This is in contrast with the negative feedback case in which square waves are always symmetric [26]. For both in- and out-of-phase square waves the period coincides with the predicted one within order $\varepsilon$. For $s_{0}=2 / 3$ the in-phase fundamental square wave corresponds to $k_{1}=2$ and $m_{1}=3$, thus $T_{1}^{\text {in }}=1 / 3$, while the fundamental out-of-phase oscillation corresponds to $k_{0}=1$ and $m_{0}=2$, thus $T_{0}^{\text {out }}=2 / 3$. We finally note that the period does not depend on the offset phase $\Phi$, thus varying the offset phase allows to tune the asymmetry of the square waves without changing the period.

We now analyze the coexistence of multiple harmonics. As discussed in Sec. III, the linear stability analysis predicts the emergence of multiple coexisting harmonics at $\chi=-1$ 

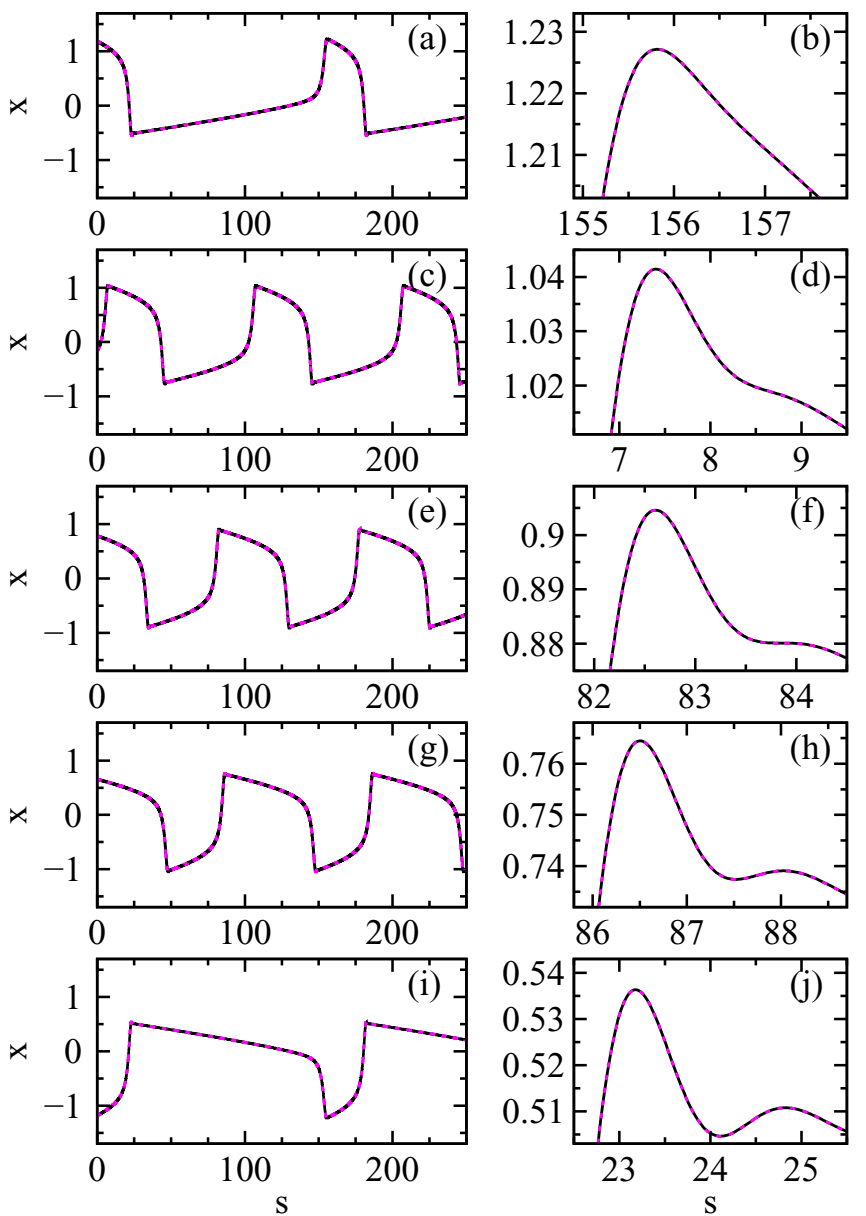

FIG. 7. (Color online) Microwave oscillations with $\gamma=0.5$, $P=1.5, T_{f}=40 \mathrm{~ns}$, and $T_{c}=60 \mathrm{~ns}$, so $s_{0}=2 / 3, \varepsilon=4.17 \times 10^{-4}$, and $\delta=0.012$. In (a) and (b) we consider $\Phi=-0.3795 \pi$, in (c) and (d) $\Phi=-0.3 \pi$, in (e) and (f) $\Phi=-0.25 \pi$, in (g) and (h) $\Phi=-0.2 \pi$, and in (i) and (j) $\Phi=-0.1205 \pi$.

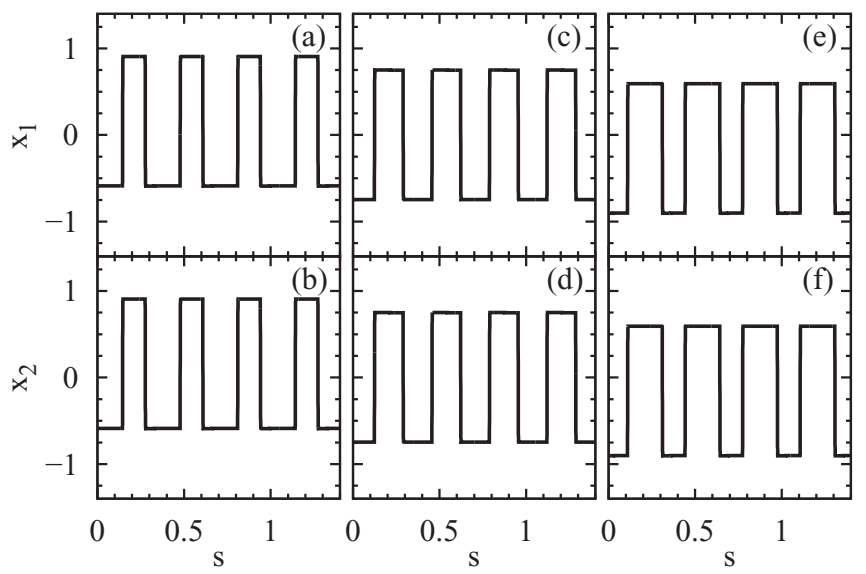

FIG. 8. In-phase fundamental square wave for $\gamma=0.5, P=1.5$, $T_{f}=40 \mathrm{~ns}$, and $T_{c}=60 \mathrm{~ns}$, so $s_{0}=2 / 3, \varepsilon=4.17 \times 10^{-4}$, and $\delta=$ 0.012 . In (a) and (b) we consider $\Phi=-0.3 \pi$, in (c) and (d) $\Phi=$ $-0.25 \pi$, and in (e) and (f) $\Phi=-0.2 \pi$. The period of this solutions is $T_{1}^{\text {in }}=1 / 3$.

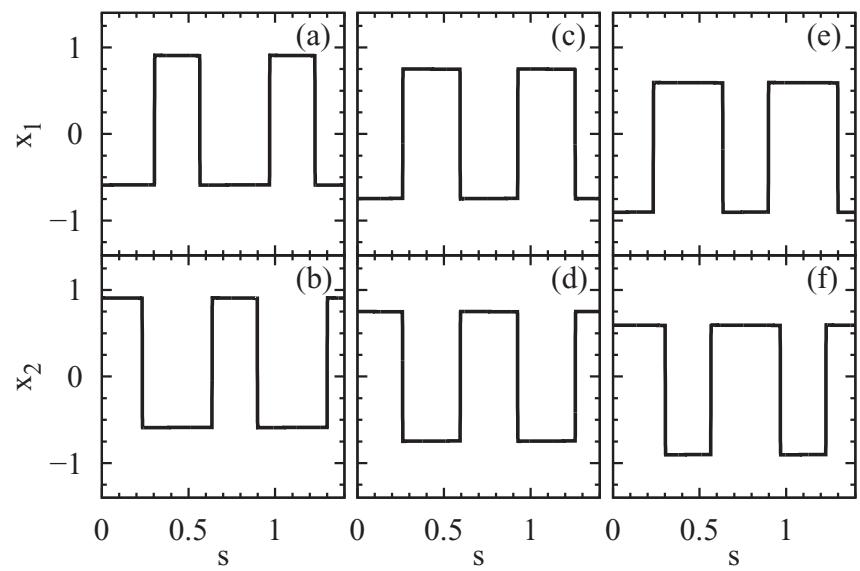

FIG. 9. Out-of-phase fundamental square wave for the same parameter values as Fig. 8 with period $T_{0}^{\text {out }}=2 / 3$.

but it does not assess the stability of these solutions. Indeed, several stable harmonics coexist for given parameters values as illustrated in Fig. 10. The different harmonics are obtained from numerical integration of the dynamical equations (5)
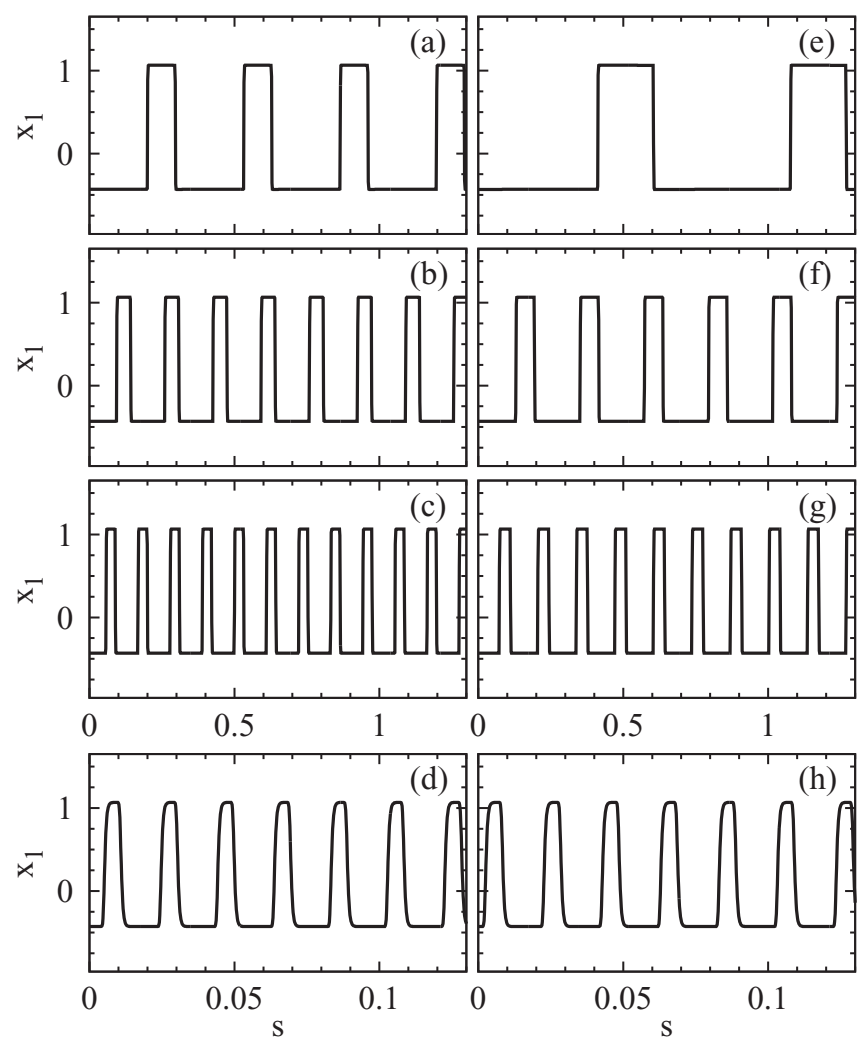

FIG. 10. Time trace of square-wave periodic solutions for the same paramaters as in Fig. 8 but where $\Phi=-0.35 \pi$. Panels (a)-(d) display coexisting in-phase solutions obtained starting with suitable initial conditions as indicated in the text. Panels (e)-(h) display coexisting out-of-phase solutions. The top row shows the fundamental solution (a)-(e). The second row shows the 1st harmonic (b)-(f). The third row shows the 2 nd harmonic (c) $-(\mathrm{g})$. The bottom row shows the 17th harmonic (d)-(h). Notice that the time scale used in panels (d) and $(\mathrm{h})$ is 10 times smaller than in the other panels. 
starting from different initial conditions. More precisely, we take as initial condition for $x_{1}(s)$ within the interval $-\max \left(1, s_{0}\right)<s<0$ a square wave with extrema $x_{a}$ and $x_{b}$, plateau lengths $s_{a}$ and $s_{b}$, and period $T_{j}^{\text {in }}$ or $T_{j}^{\text {out }}$. An analytic approximation for the values of $x_{a}, x_{b}, s_{a}$ and $s_{b}$ is derived in the next section. For in-phase solutions, the initial condition for $x_{2}$ is given by $x_{2}(s)=x_{1}(s)$, while for out-of-phase solutions we take $x_{2}(s)=x_{1}(s+T / 2)$.

Figure 10(a) shows the fundamental in-phase solution $x_{1}(s)$ with period $T_{1}^{\text {in }}=1 / 3$. The time trace for $x_{2}(s)$ (not shown) coincides with the one for $x_{1}(s)$. Figures 10(b) and 10(c) show $x_{1}(s)$ for the 1 st and 2 nd in-phase harmonics, with periods $T_{2}^{\text {in }}=T_{1}^{\text {in }} / 2=1 / 6$ and $T_{3}^{\text {in }}=T_{1}^{\text {in }} / 3=1 / 9$ respectively. Higher-order harmonics are also solutions. As an example, Fig. 10(d) shows the $17^{\text {th }}$ in-phase harmonic which has a period $T_{18}^{\text {in }}=T_{1}^{\text {in }} / 18=1 / 54$ (notice that we have used a different scale in the time axis). As regards out-of-phase solutions, the fundamental solution with period $T_{0}^{\text {out }}=2 / 3$ is shown in Fig. 10(e). As in the previous case, we only display the time traces for $x_{1}(s)$. The time traces for $x_{2}(s)$ are identical but out of phase with respect to $x_{1}(s)$, that is, $x_{2}(s)=x_{1}\left(s+T^{\text {out }} / 2\right)$. Figures 10(f) and 10(g) show the 1st and 2nd harmonics, with periods $T_{1}^{\text {out }}=T_{0}^{\text {out }} / 3=2 / 9$ and $T_{2}^{\text {out }}=T_{0}^{\text {in }} / 9=2 / 27$ respectively. Finally, Fig. 10(h) displays the $17^{\text {th }}$ harmonic, which has a period $T_{17}^{\text {out }}=T_{0}^{\text {out }} / 35=2 / 105$.

All these coexisting solutions are stable against small numerical perturbations. In the limit $\varepsilon=0$ the number of possible square-wave periodic solutions coexisting for the same parameter values is infinite. In practice, however, the number of harmonics is finite because the minimal period of the square-wave periodic solution is limited by the transition time between the plateaus of the square waves, which is of order $\varepsilon$. Nevertheless, as can be seen from Fig. 10, this system can exhibit tens of coexisting square-wave periodic solutions. In addition, for $|\chi|$ beyond $\left|\chi_{0}^{\text {in }}\right|$ the microwave in-phase solution also coexists with the multiple fast solutions. For instance, the microwave shown in Figs. 7(c) and 7(d) has been obtained with the same parameter values as the square wave shown in Figs. 8(a) and 8(b). The microwave solution turns out to have the larger basin of attraction and, starting from arbitrary initial conditions, we usually end up in this solution. However, the system may operate in any fast square-wave solution by setting the proper initial condition.

\section{ASYMMETRIC PERIODIC SQUARE-WAVE OSCILLATIONS}

In this section, we consider periodic asymmetric square waves with two different plateaus of lengths $s_{a}$ and $s_{b}$, and thus period $T=s_{a}+s_{b}$, and extrema $x_{a}>0$ and $x_{b}<0$, as illustrated in Fig. 11, and we analyze the form of the solutions in the limit of large delays. More precisely, we extend the procedure of Ref. [13] for a single OEO to coupled OEOs in order to determine the extrema of the solutions and the length of each plateau. To this end, we consider lagged solutions of the form

$$
x_{2}\left(s-1+s_{0}\right)=x_{1}(s) \equiv x(s),
$$

where the lag time $1-s_{0}$ corresponds physically to the difference $T_{c}-T_{f}$ normalized to $T_{c}$. In-phase and out-of-phase

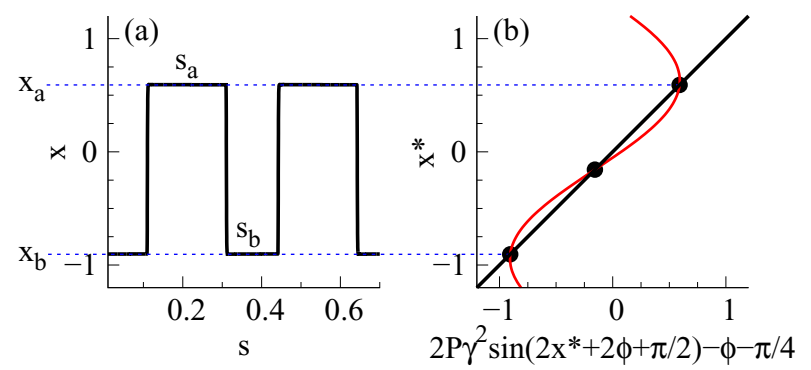

FIG. 11. (Color online) (a) In-phase numerical solution of the full dynamical model (1) for $\Phi=-0.2 \pi, \gamma=0.5, P=1.5, T_{f}=$ $40 \mathrm{~ns}$, and $T_{c}=60 \mathrm{~ns}$, so $s_{0}=2 / 3, \varepsilon=4.17 \times 10^{-4}$, and $\delta=0.012$. (b) Graphical representation of Eq. (58). The roots (dark dots) are found where the dark line [left-hand side of Eq. (58)] intersects the red curve [right-hand side of Eq. (58)]. The roots $x_{a}$ and $x_{b}$ coincide with the extrema of the numerical solution plotted in (a).

solutions are particular cases. For the in-phase oscillations Eq. (32) leads to $x_{2}(s)=x_{1}(s)$. For out-of-phase oscillations, from (34) we have $x_{2}\left(s-T^{\text {out }} / 2\right)=x_{1}(s)$, namely out-ofphase periodic solutions are delayed by half of the period.

Substitution of (39) into Eqs. (5) leads to

$$
\begin{gathered}
\varepsilon x^{\prime}(s)=-x(s)-\delta Y(s) \\
+2 P \gamma^{2}\left\{\cos \left[2 x\left(s-s_{0}\right)+2 \Phi\right]-\cos 2 \Phi\right\}, \\
Y^{\prime}(s)=x(s) .
\end{gathered}
$$

Since for both in- and out-of-phase oscillations $s_{0}$ is a multiple of the period $T$ [see Eqs. (31) and (33)], in the limit $\varepsilon \rightarrow 0$ Eq. (40) leads to

$$
\begin{gathered}
Y(s)=-\frac{x(s)}{\delta}+\frac{2 P \gamma^{2}}{\delta}\{\cos [2 x(s)+2 \Phi]-\cos (2 \Phi)\} \\
Y^{\prime}(s)=x(s)
\end{gathered}
$$

An approximate solution can be obtained taking advantage of the small value of $\delta$. Specifically, we consider the following expansion of the solution:

$$
\begin{gathered}
x(s)=x^{0}(s)+\delta x^{1}(s)+\ldots, \\
Y(s)=\delta^{-1} Y^{0}(s)+Y^{1}(s)+\ldots,
\end{gathered}
$$

where $x^{0}(s)=x_{a}$ along the positive plateau $0<s<s_{a}$ and $x^{0}(s)=x_{b}$ along the negative plateau $s_{a}<s<T=s_{b}+s_{a}$. At order $\delta^{-1}$ we have

$$
Y^{0}(s)=-x^{0}(s)+2 P \gamma^{2}\left[\cos \left(2 x^{0}(s)+2 \Phi\right)-\cos (2 \Phi)\right],
$$

$$
Y^{\prime 0}(s)=0 .
$$


At order $\delta^{0}$ we obtain

$$
\begin{gathered}
Y^{1}(s)=-x^{1}(s)\left[1+4 P \gamma^{2} \sin \left(2 x^{0}(s)+2 \Phi\right)\right], \\
Y^{\prime 1}(s)=x^{0}(s) .
\end{gathered}
$$

Since according (47) $Y^{0}(s)$ is a constant, the continuity of $Y(s)$ implies $Y^{1}\left(s_{a}^{-}\right)=Y^{1}\left(s_{a}^{+}\right)$. Then solving (49) with the initial condition $Y^{1}(0)=0$ leads to

$$
\begin{gathered}
Y^{1}(s)=x_{a} s, \quad 0 \leqslant s \leqslant s_{a}, \\
Y^{1}(s)=x_{a} s_{a}+x_{b}\left(s-s_{a}\right), \quad s_{a}<s \leqslant T .
\end{gathered}
$$

Since we are considering periodic solutions with period $T=s_{a}+s_{b}, Y^{1}\left(s_{a}+s_{b}\right)=Y^{1}(0)$, which leads to an equation relating $s_{a}, s_{b}, x_{a}$, and $x_{b}$,

$$
x_{a} s_{a}+x_{b} s_{b}=0 .
$$

Since $s_{a}>0$ and $s_{b}>0, x_{a}$ and $x_{b}$ must have a different sign. Without losing generality, we consider $x_{a}>0$ and $x_{b}<0$.

Substituting (50) and (51) into (48) we obtain

$$
\begin{aligned}
& x^{1}(s)=-\frac{x_{a} s}{1+4 P \gamma^{2} \sin \left(2 x_{a}+2 \Phi\right)}, 0 \leqslant s \leqslant s_{a}, \\
& x^{1}(s)=-\frac{x_{a} s_{a}+x_{b}\left(s-s_{a}\right)}{1+4 P \gamma^{2} \sin \left(2 x_{b}+2 \Phi\right)}, \quad s_{a}<s \leqslant T .
\end{aligned}
$$

The continuity condition $x^{1}\left(s_{a}^{+}\right)=x^{1}\left(s_{a}^{-}\right)$implies

$$
\cos \left(x_{b}+x_{a}+2 \Phi\right) \sin \left(x_{b}-x_{a}\right)=0 .
$$

Since $x_{a} \neq x_{b}$, the solution of (55) is $x_{a}+x_{b}+2 \Phi= \pm(2 n+$ 1) $\pi / 2$. For $\Phi \in[-\pi / 2,0]$, as suitable for negative feedback, we obtain the following equation relating $\Phi$ to the extrema $x_{a}$ and $x_{b}$

$$
\Phi=-\pi / 4-\left(x_{a}+x_{b}\right) / 2 .
$$

Therefore the square wave is symmetric $\left(x_{b}=-x_{a}\right)$ for $\Phi=$ $-\pi / 4$, asymmetric with $x_{a}>\left|x_{b}\right|$ for $\pi / 2 \leqslant \Phi<-\pi / 4$, and asymmetric with $x_{a}<\left|x_{b}\right|$ if $-\pi / 4<\Phi<0$. To determine $x_{a}$ and $x_{b}$ we need an additional equation relating both. Subtracting Eq. (46) with $x^{0}(s)=x_{a}$ from the same equation with $x^{0}(s)=x_{b}$ we obtain

$$
\left(x_{a}-x_{b}\right)=4 P \gamma^{2} \sin \left(x_{a}+x_{b}+2 \Phi\right) \sin \left(x_{b}-x_{a}\right) .
$$

Introducing (56) into (57) we obtain an equation for $x_{a}$ or $x_{b}$ which we label as $x^{*}$,

$$
x^{*}=2 P \gamma^{2} \sin \left(2 x^{*}+2 \Phi+\pi / 2\right)-\Phi-\pi / 4 .
$$

Introducing (58) into (46) we get the value for $Y_{0}$,

$$
Y^{0}=\Phi+\pi / 4-2 P \gamma^{2} \cos (2 \Phi) \text {. }
$$

Defining

$$
z^{*}=x^{*}+\Phi+\pi / 4
$$

Eq. (58) can be written as

$$
z^{*}=2 P \gamma^{2} \sin \left(2 z^{*}\right) \text {. }
$$

Equation (61) has always the root $z^{*}=0$, which corresponds to $x^{*}=x_{c}=-\pi / 4-\Phi$. Substituting this result into (56) we get that $x_{c}=\left(x_{a}+x_{b}\right) / 2$, namely this root corresponds to the

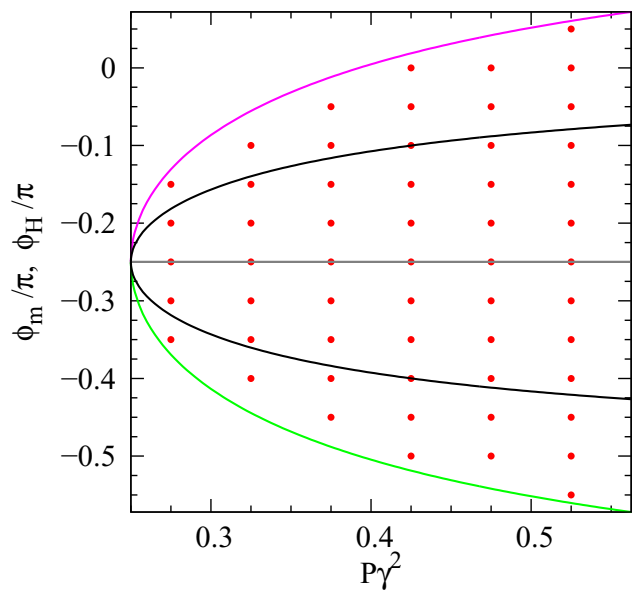

FIG. 12. (Color online) Red dots show the region of existence of the square-wave solutions. Boundaries are set by the solutions of Eq. (62) $\Phi_{m_{a}}$ [pink (dark gray) line] and $\Phi_{m_{b}}$ [green (light gray) line] which correspond to the most asymmetric square waves. Black lines correspond to the Hopf bifurcation. Square waves coexist with the zero solution in the subcritical region between black and color lines.

average of the two extrema of the square wave. For $P \gamma^{2}>1 / 4$ there are also two nonzero roots, $z_{a}$ and $z_{b}$ with $z_{b}=-z_{a}$, which correspond to the extrema of the square wave $x_{a}>$ 0 and $x_{b}<0$. For $\Phi=-\pi / 4, x^{*}=z^{*}$ and $x_{b}=-x_{a}$. As the offset phase departs from $\Phi=-\pi / 4$, the roots for $x$ are asymmetrically located with respect to 0 . The limit value of $\Phi$ for which Eq. (58) has solutions with $x_{a}>0$ and $x_{b}<0$ can be obtained by setting $x^{*}=0$ and solving the resulting equation,

$$
\Phi_{m}=-\pi / 4-2 P \gamma^{2} \cos \left(2 \Phi_{m}\right) .
$$

This equation has a trivial solution $\Phi_{m_{s}}=-\pi / 4$ and two nontrivial solutions $\Phi_{m_{a}}$ and $\Phi_{m_{b}}$ which are plotted in Fig. 12 as a function of $P \gamma^{2}$. For a given value of $P \gamma^{2}, \Phi_{m_{a}}$ corresponds to the most asymmetric solution with $x_{a}=0$ and $s_{a}=T$ [pink (dark gray) line] and $\Phi_{m_{b}}$ to the one with $x_{b}=0$ and $s_{b}=T$ [green (light gray) line]. In contrast, the symmetric solution $\Phi_{m_{s}}$ is given by the horizontal line.

Since (61) is independent of $\Phi$, using (60) it turns out that, for a given $P \gamma, x_{a}$, and $x_{b}$ have a linear dependence with $\Phi$ with slope -1 . Then, since the intercept is given by $\Phi_{m_{a}}$ or $\Phi_{m_{b}}$, we can write

$$
\begin{aligned}
& x_{a}=-\Phi+\Phi_{m_{a}}, \\
& x_{b}=-\Phi+\Phi_{m_{b}} .
\end{aligned}
$$

Comparing these equations with (56) we have $\Phi_{m_{a}}+\Phi_{m_{b}}=$ $-\pi / 2$. The range of offset phases $\left[\Phi_{m_{b}}, \Phi_{m_{a}}\right]$ for which asymmetric periodic square waves are predicted to exist increases with $P \gamma^{2}$. For comparison the black lines show the offset phase at the Hopf bifurcation $\chi_{n}=-1$, which, from Eq. (8), is given by $\Phi_{\mathrm{H}}=(-1)^{k-1} \frac{1}{2} \arcsin \left(\frac{1}{4 P \gamma^{2}}\right)+k \pi$. It is clear that for a given $P \gamma^{2}$ the range $\left[\Phi_{m_{b}}, \Phi_{m_{a}}\right]$ extends well beyond the range given by Hopf offset phases. Therefore according to this theoretical analysis the Hopf bifurcation leading to square waves is subcritical for offset phases that differ from $-\pi / 4$. In 
fact, for $P \gamma^{2}=\pi / 8$ asymmetric solutions exist over the whole range of positive feedback with $\Phi \in[-\pi / 2,0]$ and for larger $P \gamma^{2}$ they can be found beyond this range. Thus, although asymmetric square waves are born at Hopf bifurcations which can only take place for positive feedback, once they are born increasing $P \gamma^{2}$ and changing the feedback phase we can obtain stable asymmetric square waves with negative feedback.

Finally, taking into account that the total period is $T=s_{a}+$ $s_{b}$, the length of the plateaus can be obtained from Eq. (52):

$$
\begin{gathered}
s_{a}=-\frac{x_{b}}{x_{a}-x_{b}} T, \\
s_{b}=\frac{x_{a}}{x_{a}-x_{b}} T .
\end{gathered}
$$

Since $x_{a}$ and $x_{b}$ are the roots of (58) and therefore depend on the offset-phase $\Phi$, the duty cycle of the square wave can be tuned with $\Phi$ as seen from numerical simulations in Sec. IV and Sec. VI.

\section{COMPARISON BETWEEN THEORETICAL RESULTS FOR SQUARE WAVES AND NUMERICAL SIMULATIONS}

In this section we compare the theoretical predictions of the previous section with numerical simulations of Eq. (5). The first feature to note is that, comparing Fig. 8 with Fig. 9, it is clear that for a given value of $\chi$ both extrema $x_{a}$ and $x_{b}$ are the same for in- and out-of-phase oscillations, as predicted by Eq. (58) which is the same for in- and out-of-phase oscillations.

A more detailed comparison of the theoretical predictions and numerical results is shown in Fig. 13 for $P=1.5$ and $\gamma=0.5$ as function of the offset phase $\Phi$. Figure 13(a) shows the bifurcation diagram of the extrema $x_{a}$ and $x_{b}$. The pink (dark gray) and green (light gray) solid lines represent, respectively, the theoretical values for $x_{a}$ and $x_{b}$ obtained solving Eq. (58), while the solid black line shows the stable zero solution. Unstable solutions are plotted as dotted black lines. The unstable branch of periodic solutions which connects the Hopf bifurcation point to the branch of stable periodic square-wave solutions has been obtained using DDE-BIFTOOL [29-32]. The results of numerical simulations are plotted using symbols. Red points correspond to in- and out-of-phase square-wave obtained starting from a suitable initial condition as explained in Sec. IV. Black points represent numerical simulations verifying the stability of the zero solution. Clearly, the theoretical prediction for the square-wave amplitudes is in perfect agreement with the numerical simulations. Following Eqs. (63) and (64), the amplitudes have a linear dependence with the offset phase. The amplitudes are symmetrical for $\Phi=-\pi / 4$ and become progressively more asymmetric as $\Phi$ is moved away from this value. The square-wave solution disappears when one of the amplitudes touches zero. Starting from an offset phase far away from the symmetric case $\Phi=-\pi / 4$ and changing $\Phi$ towards the symmetric value, the bifurcation from the zero solution to the square-wave solutions appears subcritically, as predicted theoretically in Sec. V. The width of the subcritical region agrees with the theoretical prediction. We note that for smaller values of $P \gamma^{2}$ close to the limit value 0.25 numerically one finds that the subcritical region is smaller than the predicted one because at these power levels nanosecond solutions do not have a square-wave shape.

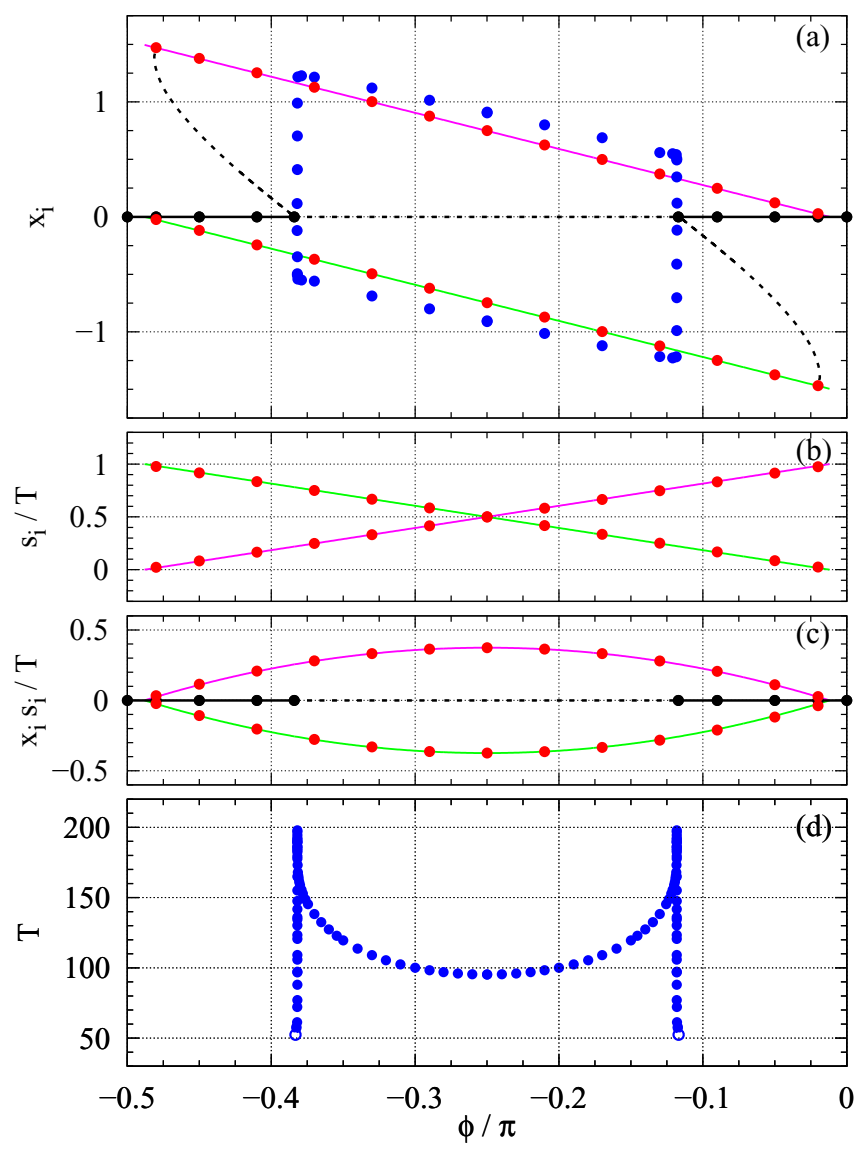

FIG. 13. (Color online) Bifurcation diagram of the zero solution showing (a) the extrema $x_{a}$ [pink (dark gray) line] and $x_{b}$ [green (light gray) line] of the square waves, (b) the length $s_{1}$ [pink (dark gray) line] and $s_{2}$ [green (light gray) of the plateaus scaled to the period $T$, and (c) the scaled products $x_{a} s_{a} / T$ and $x_{b} s_{b} / T$ as a function of $\Phi$ with $P=1.5$ and $\gamma=0.5$. Panel (d) shows the period of microwaves. Lines correspond to the theoretical prediction of stable (solid) or unstable (dashed) solutions (see text). Filled circles correspond to the numerical integration of the full dynamical model with $T_{f}=$ $40 \mathrm{~ns}$ and $T_{c}=60 \mathrm{~ns}$ leading to different solutions depending on $\Phi$ and the initial condition: zero solution (black points), in- or outof-phase square waves (see text) (red points), and microwaves (blue points). The empty blue circles in (d) show the theoretical threshold for microwaves.

Figure 13(b) shows the lengths $s_{a}$ and $s_{b}$ of the two plateaus of the asymmetric square waves as predicted by Eq. (65) and Eq. (66) and scaled to the period $T$, while Fig. 13(c) shows the scaled products $x_{a} s_{a} / T$ and $x_{b} s_{b} / T$. Again the theoretical prediction is in excellent agreement with the numerical simulations.

For comparison, blue points in Fig. 13(a) show the amplitude from numerical simulations of the microwave solution that coexists with the nanosecond square waves. Microwaves are born supercritically from the zero solution, an indication that they have a nature that differs from that of the fast nanosecond square waves. Furthermore, as indicated when discussing Fig. 7, the microwave period has a strong dependence with $\Phi$. Quantitative results for this dependence are shown in Fig. 13(d). Empty circles in Fig. 13(d) correspond 


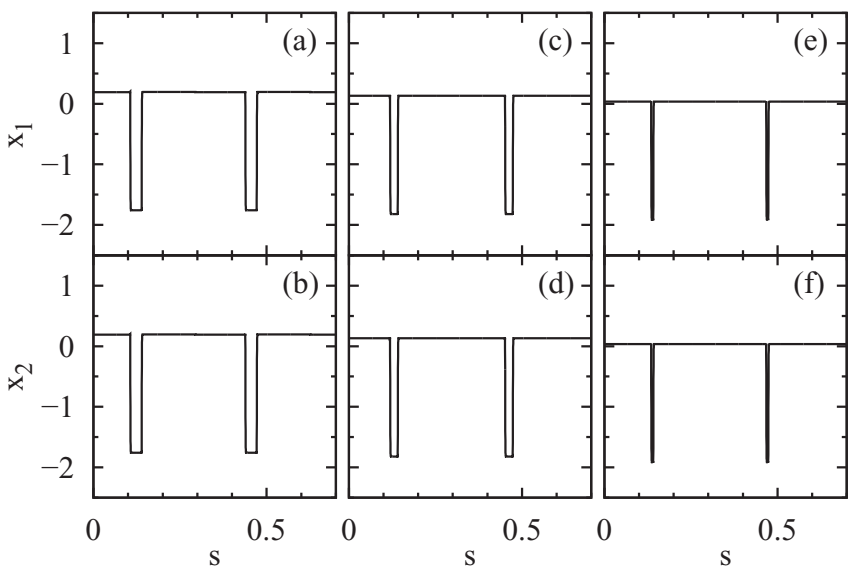

FIG. 14. Periodic square waves with $P=2.1, \gamma=0.5, T_{f}=$ $40 \mathrm{~ns}, T_{c}=60 \mathrm{~ns}, \chi=0[(\mathrm{a})$ and (b)], $\chi=0.02[(\mathrm{c})$ and (d)], and $\chi=0.05[(\mathrm{e})$ and (f) $]$.

to the theoretical prediction for the period at threshold, $T\left(\chi_{0}^{\text {in }}\right)$. Just after threshold, the stationary amplitudes $x_{a}$ and $\left|x_{b}\right|$ and the period increase very fast. The period increases by a factor 4 from $\Phi=-0.3826 \pi(\chi=-1.0090)$ to $\Phi=-0.3818 \pi(\chi=$ $-1.0143)$ where it takes a maximum. Both amplitudes $x_{a}$ and $\left|x_{b}\right|$ keep increasing until $\Phi_{\max }=-0.379 \pi(\chi=-1.0337)$ where $x_{a}$ reaches a maximum. At this point the amplitudes are larger than those of the square wave for the same parameters. After that, increasing $\Phi, x_{a}$, and $x_{b}$ decrease practically linearly with $\Phi$ with a slope slightly smaller than that of the square waves. At $\Phi=-0.25 \pi$, where the microwave is symmetrical, the period has a relative minimum taking a value which is, nevertheless, about the double that of the one at threshold. The value of the period as function of the feedback phase is symmetrical with respect to $\Phi=-0.25 \pi$. As for the amplitudes, $x_{a}$ and $x_{b}$ keep decreasing linearly up to $\Phi_{\min }=-0.121 \pi(\chi=-1.0337)$, where $x_{b}$ reaches a minimum beyond which $x_{a}$ and $\left|x_{b}\right|$ decrease fast to zero.

Increasing $P \gamma^{2}$ the bifurcation diagram has a similar shape but the range in which oscillatory solutions exist increases. As predicted by (62) for periodic square waves, for $P \gamma^{2}>\pi / 8$ this range includes positive values of $\Phi$ (negative feedback).
This theoretical prediction is confirmed by numerical simulations, as illustrated in Fig. 14 which displays in-phase asymmetric solutions with zero feedback $(\chi=0)$ and negative feedback $(\chi>0)$ obtained from numerical integration of (5). In Fig. 14 the positive plateau is larger than the negative plateau. In general, for any solution with $\Phi \in[-\pi / 4, \pi / 4]$ the positive plateau is longer than the negative one, while for $\Phi \in[-3 \pi / 4,-\pi / 4]$ is the other way around.

If the laser power is further increased, the square-wave periodic solutions degrade and finally destabilize. The system becomes chaotic although $x_{1}$ and $x_{2}$ can be still synchronized for moderate values of the laser power, as it can be seen in Figs. 15(a) and 15(b), 15(c) and 15(d), and 15(e) and 15(f). For $P \approx 2.9$ synchronization is lost and for larger $P$ the solutions become more and more chaotic, as can be seen in Figs. 15(g) and 15(h), 15(i) and 15(j), and 15(k) and 15(l).

\section{MISMATCH IN THE DELAY TIMES}

The asymmetric square-wave solutions emerging from a Hopf bifurcation with positive feedback are stable to numerical perturbations and robust to changes of the offset phase. Nevertheless, from the experimental point of view it is important to assess also the robustness in front of an eventual mismatch in the delay times. In this section we analyze the effect of a small delay-time mismatch keeping $T_{c}$ fixed while $T_{f}$ is changed.

In Fig. 16 we can see the fundamental (a) and first harmonic (b) out-of-phase periodic square waves generated for a perfect matching condition $s_{0}=2$. For a small mismatch in $T_{f}$, the plateaus become shorter and the transition looses sharpness, as can be seen in Figs. 16(c) and 16(d), 16(e) and 16(f), and 16(g) and $16(\mathrm{~h})$ for 1,3 , and $5 \%$ mismatch in $T_{f}$, respectively. The fundamental solution is robust to mismatches larger than 5\%, but the first harmonic is more sensible to mismatch because it has a smaller period. The same effect takes place for in-phase solutions, as can be seen from Fig. 17, although the first harmonic in this case only survives until there is $3 \%$ mismatch. For 5\% mismatch the first harmonic initial condition leads to a stationary solution with a much smaller period $(T=0.083)$ [Fig. 17(h)]. For the same parameter values out-of-phase solutions are more resilient to delay time mismatches because

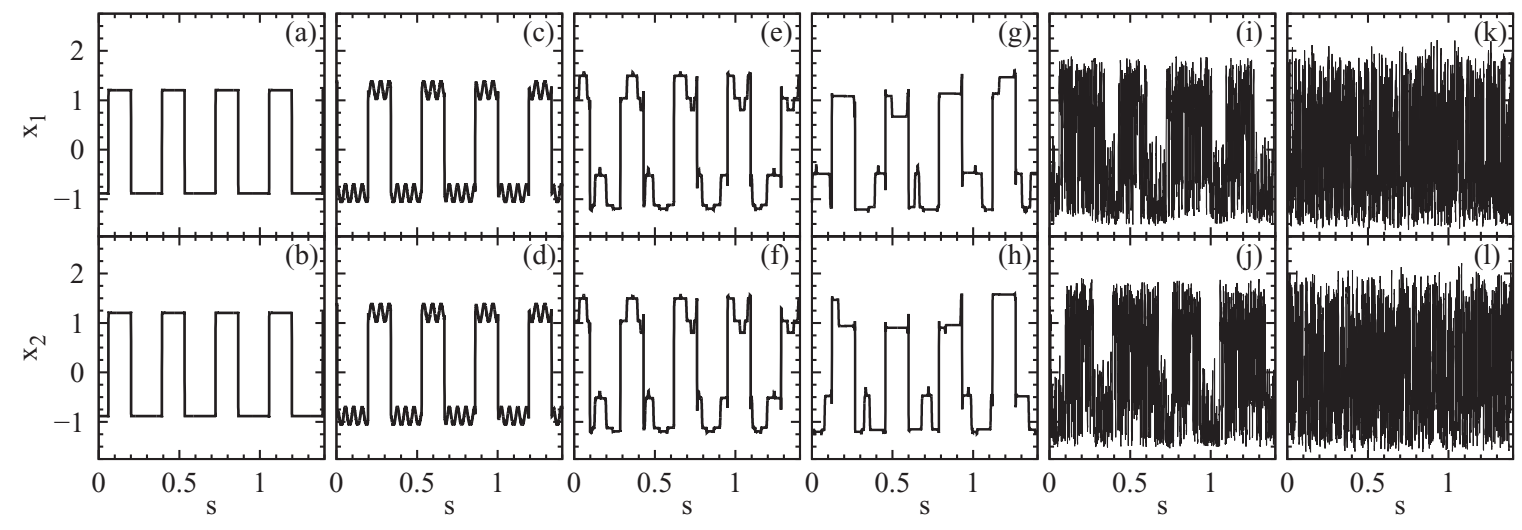

FIG. 15. Numerical simulations with $\gamma=0.5, T_{f}=40 \mathrm{~ns}, T_{c}=60 \mathrm{~ns}$, and $\Phi=-0.3 \pi$ and where the initial condition is an in-phase periodic solution with period $T_{\text {in }}=2 / 3$. In (a) and (b) we consider $P=2.4$, in (c) and (d) $P=2.5$, in (e) and (f) $P=2.8$, in (g) and (h) $P=2.9$, in (i) and (j) $P=3.5$, and in (k) and (l) $P=4$. 

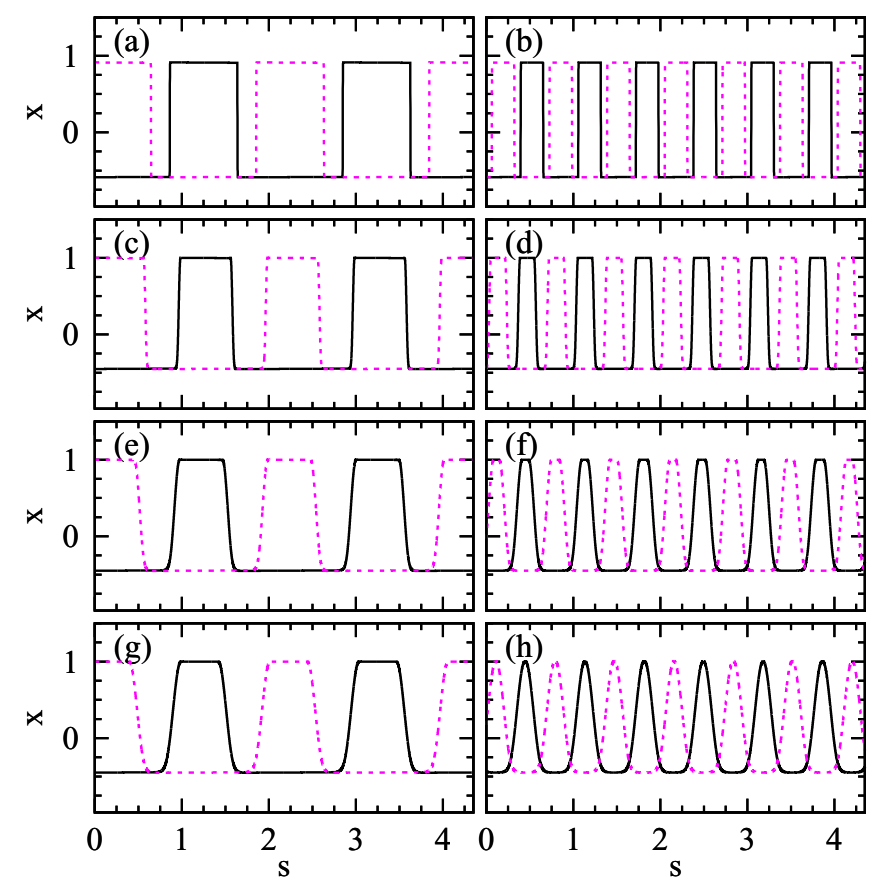

FIG. 16. (Color online) Out-of-phase periodic solutions with $\Phi=-0.3 \pi, \gamma=0.5, P=1.5, T_{c}=30 \mathrm{~ns}$, so $\varepsilon=8.33 \times 10^{-4}$ and $\delta=0.006$, with perfect matching condition $s_{0}=2$ with $T_{f}=60 \mathrm{~ns}$ [(a) and (b)], $1 \%$ mismatch with $T_{f}=60.6 \mathrm{~ns}$ [(c) and (d)], $3 \%$ mismatch with $T_{f}=61.8 \mathrm{~ns}$ [(e) and (f)], and 5\% mismatch with $T_{f}=62.7 \mathrm{~ns}[(\mathrm{~g})$ and (h)]. Panels (a), (c), (e), and (g) show the fundamental solution (with period $T_{0}^{\text {out }}=2$ ); panels (b), (d), (f), and (h) show the first harmonic (with period $T_{1}^{\text {out }}=2 / 3$ ).
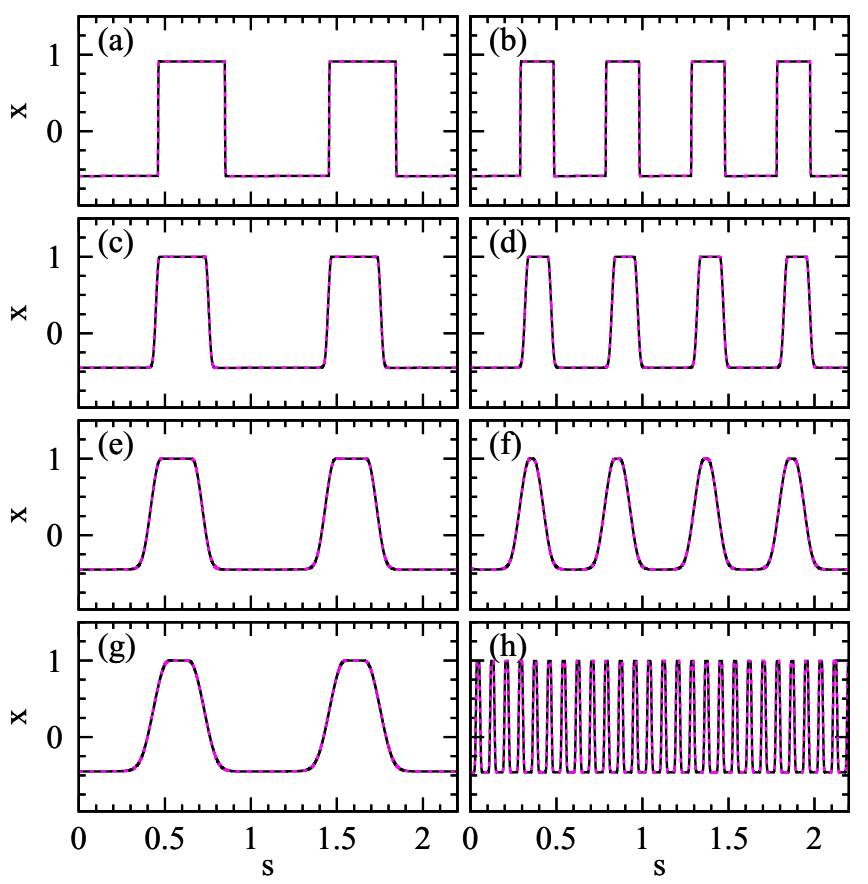

FIG. 17. (Color online) In-phase periodic solutions for the same parameter values as Fig. 16. Panels (a), (c), (e), and (g) show the fundamental nanosecond solution (with period $T_{1}^{\text {in }}=1$ ) while panels (b), (d), and (f) show the first harmonic (with period $T_{2}^{\text {in }}=0.5$ ). Panel (h) shows the stationary solution found with $5 \%$ mismatch when the initial condition is the first harmonic.
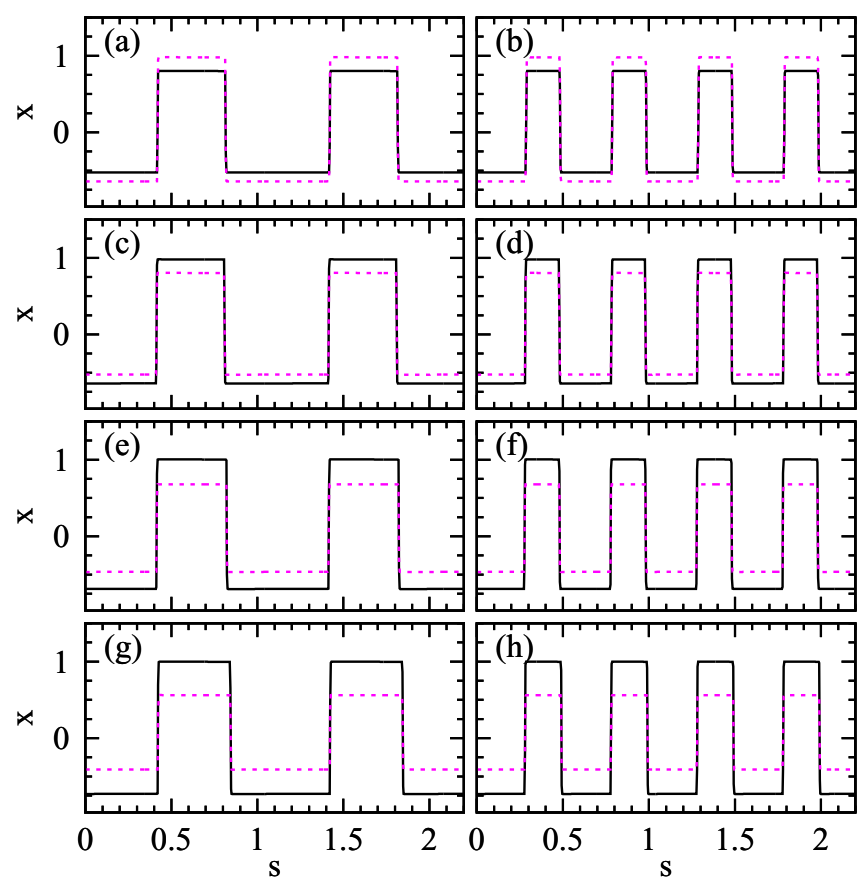

FIG. 18. (Color online) In-phase periodic solutions with $\Phi=$ $-0.3 \pi, P=1.5, T_{f}=60 \mathrm{~ns}$, and $T_{c}=30 \mathrm{~ns}$, so $s_{0}=2, \varepsilon=$ $8.33 \times 10^{-4}$, and $\delta=0.006$ with $\gamma_{11}=\gamma_{12}=0.5$ while increasing the coupling coefficient $\gamma_{21}=1-\gamma_{22}: \gamma_{21}=0.45$ (a) and (b), $\gamma_{21}=$ $0.55(\mathrm{c})$ and (d), $\gamma_{21}=0.6(\mathrm{e})$ and (f), and $\gamma_{21}=0.65(\mathrm{~g})$ and $(\mathrm{h})$. The solid black line corresponds to $x_{1}$ and the red dashed line to $x_{2}$. Panels (a), (c), (e), and (g) display the fundamental square wave (with period $T=1$ ) while panels (b), (d), (f), and (h) show the first harmonic.

they have a larger period, as can be seen in Fig. 3. Another way to increase the robustness to mismatch in the delay times consists of decreasing the asymmetry of the solution, since the mismatch makes the lengths of the plateaus smaller.

We recall that for symmetric square waves arising in the negative feedback case, at $2 \%$ mismatch the degradation leads to the apparition of small secondary plateaus [26] at intermediate values of $x_{1}$ and $x_{2}$. These plateaus were not symmetrically located around $x=0$ and thus it was a way for the system to break the symmetry of the solution. On the contrary, asymmetric square waves show a larger degree of robustness since they do not exhibit secondary plateaus with a small mismatch in the delay times. Their intrinsic asymmetry allows them to flexibly adapt to a small mismatch in the delay time with a minimal deformation in the slope.

\section{MISMATCH IN THE FEEDBACK STRENGTH COEFFICIENTS}

So far we have considered that self- and cross-feedback strength coefficients are all identical, $\gamma_{i j}=\gamma$. Here we show that in- and out-of phase square-wave solutions encountered in previous sections also appear when the feedback strength coefficients differ.

In Fig. 18 we show the fundamental and first-harmonic in-phase square waves arising for $\gamma_{11}=\gamma_{12}=0.5$ and the increasing values of $\gamma_{21}$ while keeping constant $\gamma_{22}+\gamma_{21}=1$. It is clear that square waves remain stable with the same shape 


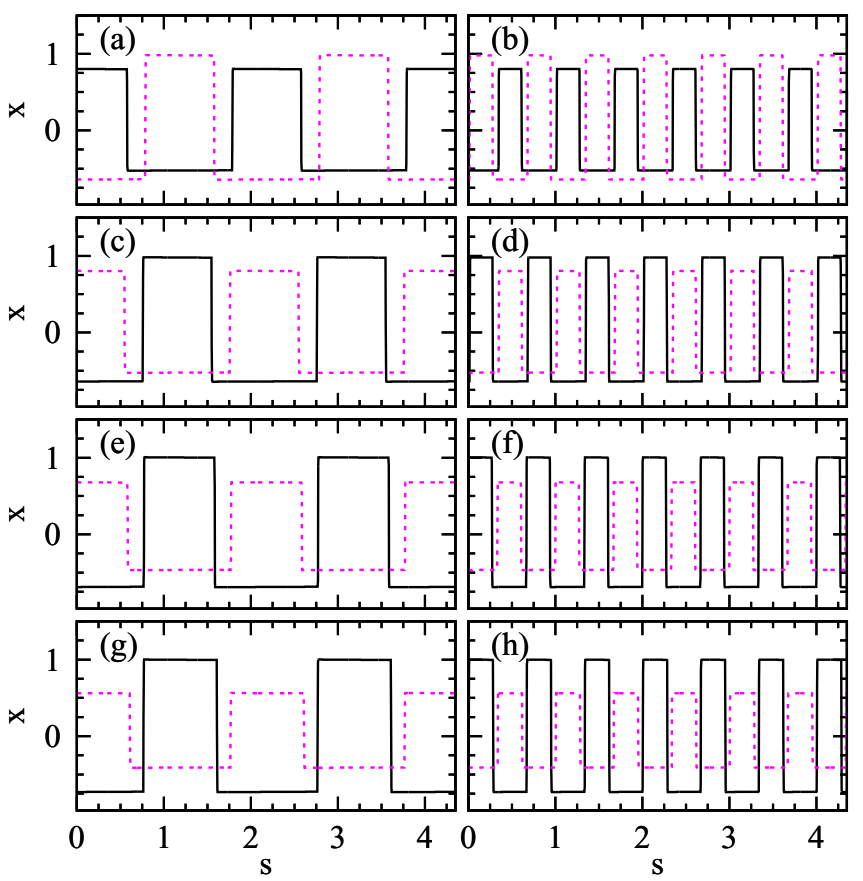

FIG. 19. (Color online) Out-of-phase periodic solutions for the same parameters as in Fig. 18 while increasing the coupling coefficient $\gamma_{21}=1-\gamma_{22}: \gamma_{21}=0.45$ [(a) and (b)], $\gamma_{21}=0.55$ [(c) and (d)], $\gamma_{21}=0.6$ [(e) and (f)], and $\gamma_{21}=0.65$ [(g) and (h)]. Panels (a), (c), (e), and (g) display the fundamental square wave (with period $T=2$ ) while panels (b), (d), (f), and (h) show the first harmonic.

and period but the amplitude of $x_{1}$ and $x_{2}$ now differs. For $\gamma_{21}<0.5$ the strength of the cross-feedback arriving at OEO $2\left(\gamma_{12}\right)$ is larger than the one arriving at OEO $1\left(\gamma_{21}\right)$ and, at the same time, the strength of the self-feedback in OEO 2 is larger than the self-feedback in OEO $1\left(\gamma_{22}>\gamma_{11}=0.5\right)$. Altogether OEO 2 has a stronger feedback than OEO 1 and, as a consequence, the amplitude of $x_{2}$ is larger. On the contrary, for $\gamma_{21}>0.5$ the strength of the cross-feedback arriving at OEO 2 is smaller than the one at OEO $1\left(\gamma_{21}>\gamma_{12}\right)$ and the self-feedback in OEO 2 is also weaker than in OEO 1 , so $x_{2}$ has a smaller amplitude than $x_{1}$. Similar results can be obtained for the out-of-phase square-wave solutions as shown in Fig. 19.

Finally, we study the effect of mismatch in the feedback strength coefficients in the microwave periodic solutions. As can be seen in Fig. 20, if the mismatch is not too large, synchronized microwave oscillations are stable. The mismatch in the feedback strength coefficients leads to an asymmetry on the feedback strength arriving at each OEO and, as a consequence, the amplitude of $x_{1}$ and $x_{2}$ differs. If the mismatch is large, microwave oscillations can develop fast oscillations on top of the part of the period with larger amplitude as shown in Figs. 20(m)-20(o). These fast oscillations are more prominent when the strong mismatch is in the direction of having a large self-feedback $\left(\gamma_{22}=0.9\right.$ and $\left.\gamma_{21}=0.1\right)$ than when it is in the opposite way $\left(\gamma_{22}=0.1\right.$ and $\left.\gamma_{21}=0.9\right)$ [compare Figs. 20(a)-20(c) with 20(m)-20(o)]. These fast oscillations are reminiscent of those encountered for a single OEO as the pump is increased leading to chaotic breathers [12,28]. Fast oscillations appear typically only in one of the plateaus, except
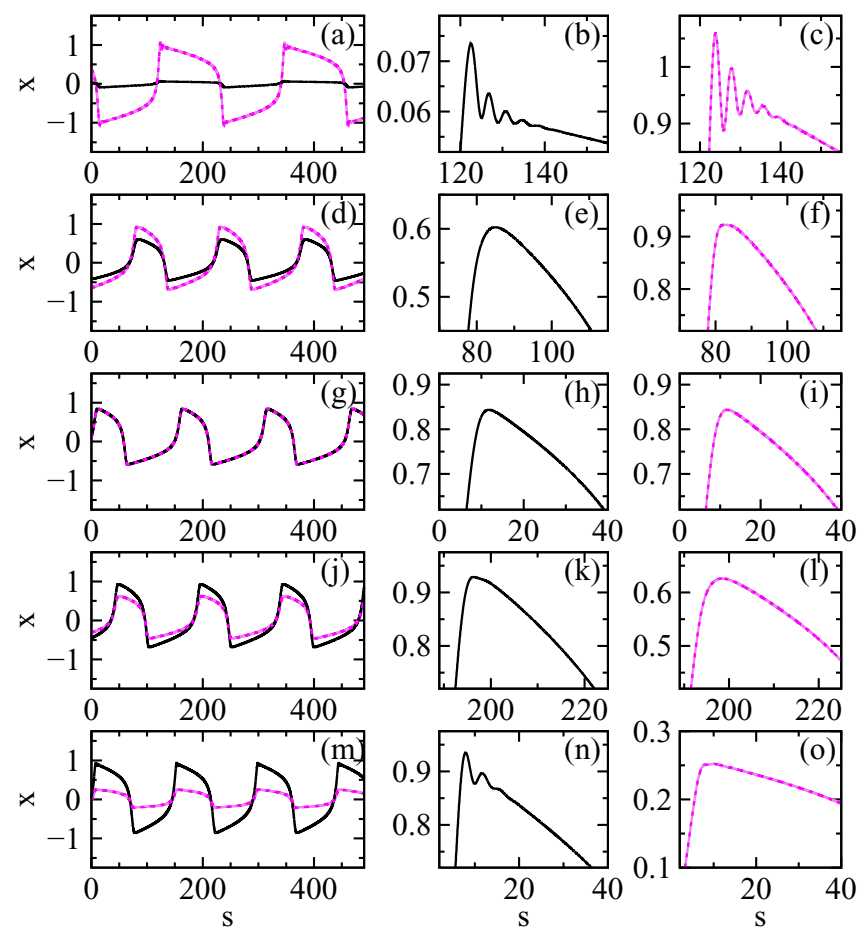

FIG. 20. (Color online) Microwave oscillations with $\Phi=$ $-0.3 \pi, P=1.3, T_{f}=60 \mathrm{~ns}$, and $T_{c}=30 \mathrm{~ns}$, so $s_{0}=2, \varepsilon=8.33 \times$ $10^{-4}$, and $\delta=0.006$, with $\gamma_{11}=\gamma_{12}=0.5$ while increasing the coupling coefficient $\gamma_{21}=1-\gamma_{22}: \gamma_{21}=0.1$ [(a)-(c)], $\gamma_{21}=0.4$ $[(\mathrm{d})-(\mathrm{f})], \quad \gamma_{21}=0.5[(\mathrm{~g})-(\mathrm{i})], \quad \gamma_{21}=0.6[(\mathrm{j})-(\mathrm{l})]$, and $\gamma_{21}=0.9$ [(m)-(o)]. Panels (e), (f), (h), (i), (k), (l), (n), and (o) are zooms of the panels on the left.

for the particular value of the offset phase $\Phi=-0.25 \pi$ for which the microwaves are symmetric. Finally, we note that the period and the duty cycle of the microwave pulses also change with the mismatch of the feedback strength coefficients. In particular, increasing the asymmetry of the coupling the period increases and the duty cycle tends to be half of the period.

\section{SUMMARY AND CONCLUDING REMARKS}

In this article we have studied analytically and numerically the dynamics of two delay coupled identical OEOs subject to positive delay feedback. We have determined the conditions for in- and out-of-phase synchronized periodic solutions and derived analytical expressions for the period of the solutions as well for the amplitude and the plateau lengths of the squarewave solutions. Our analytical results are in good agreement with numerical simulations of the full dynamical model and continuation techniques.

In comparison to the negative feedback case, $\chi>0$, the positive feedback case, $\chi<0$, shows a much richer scenario. In both cases in- and out-of-phase fast square-wave oscillations are encountered when the feedback and the coupling delay times satisfy appropriate ratios. Also, in both cases the fast square waves are born at Hopf bifurcations from the zero state at $|\chi|=1$ and multiple stable periodic solutions with different periods can coexist for the same parameter values. However, the square waves born for negative feedback are always symmetric while for positive feedback we have in general 
asymmetric square waves whose degree of asymmetry depends on the offset phase. For the negative feedback case, symmetric in-phase square waves never coexist with out-of-phase square waves. On the contrary, for positive feedback out-of-phase square waves always coexist with in-phase square waves. This provides a large degree of flexibility for tuning the period of the square waves without changing any parameter just by selecting a suitable initial condition, making this system a good candidate to be implemented for information encoding as a high-capacity memory device. Besides, the duty cycle of the periodic square waves can be tuned with the offset phase.

Curiously enough we have also shown that in fact for sufficiently large pump strength, asymmetric in- and out-ofphase square waves can also exist for negative feedback. These asymmetric square waves are born at a Hopf bifurcation taken place at positive feedback and therefore a convenient way to generate them is to start from positive feedback, change the phase, and increase the pump level.

Another difference with the negative feedback case is that for positive feedback besides the fast square-wave oscillations, which for typical parameter values are at nanosecond time scale, there are in-phase microwave periodic solutions. For the periodic microwaves both the period and the asymmetry can be tuned with the offset phase.
Finally, asymmetric square waves born at positive feedback turn out to be more robust and flexible with respect to parameter changes than the symmetric solutions obtained with negative feedback. In particular, while symmetric square waves with $\chi>0$ are quite rigid and undergo a period doubling route to chaos changing the feedback phase, asymmetric square waves are able to maintain their period by adapting their shape in response to changes of the offset phase instead of undergoing a period doubling. Furthermore, asymmetric square waves are also more robust to mismatches in the delay times with respect to the ideal ratio.

\section{ACKNOWLEDGMENTS}

This work benefited from the financial support of Ministerio de Economía y Competitividad (Spain) and Fondo Europeo de Desarrollo Regional under Projects No. FIS2012-30634 (INTENSE@COSYP) and No. TEC2012-36335 (TRIPHOP); European Social Fund and Govern de les Illes Balears under programs Grups Competitius and Formació de Personal Investigador; Fonds de la Recherche Scientifique-FNRS (Belgium); and the Belgian Science Policy Office under Grant No. IAP-7/35 "photonics@be." J.M. thanks Lionel Weicker for providing useful computer code for DDE-BIFTOOL.
[1] T. Erneux, Applied Delay Differential Equations (Springer, New York, 2000).

[2] B. Sartorius, C. Bornholdt, O. Brox, H. Ehrke, D. Hoffmann, R. Ludwig, and M. Mohrle, Electron. Lett. 34, 1664 (1998).

[3] S. Ura, S. Shoda, K. Nishio, and Y. Awatsuji, Opt. Express 19, 236833 (2011).

[4] A. Gavrielides, T. Erneux, D. W. Sukow, G. Burner, T. McLachlan, J. Miller, and J. Amonette, Opt. Lett. 31, 2006 (2006).

[5] D. W. Sukow, A. Gavrielides, T. Erneux, B. Mooneyham, K. Lee, J. McKay, and J. Davis, Phys. Rev. E 81, 025206(R) (2010).

[6] C. Masoller, D. Sukow, A. Gavrielides, and M. Sciamanna, Phys. Rev. A 84, 023838 (2011).

[7] E. Viktorov, A. Yacomotti, and P. Mandel, J. Opt. B: Quantum Semiclass. Opt. 6, L9 (2004).

[8] J. Mulet, M. Giudici, J. Javaloyes, and S. Balle, Phys. Rev. A 76, 043801 (2007).

[9] L. Mashal, G. V. der Sande, L. Gelens, J. Danckaert, and G. Verschaffelt, Opt. Express 20, 22503 (2012).

[10] X. Zhang, C. Gu, G. Chen, B. Sun, L. Xu, A. Wang, and H. Ming, Opt. Lett. 37, 1334 (2012).

[11] M. Marconi, J. Javaloyes, S. Barland, M. Giudici, and S. Balle, Phys. Rev. A 87, 013827 (2013).

[12] Y.C. Kouomou, P. Colet, L. Larger, and N. Gastaud, Phys. Rev. Lett. 95, 203903 (2005).

[13] L. Weicker, T. Erneux, O. d'Huys, J. Danckaert, M. Jacquot, Y. Chembo, and L. Larger, Phys. Rev. E 86, 055201(R) (2012); Phil. Trans. R. Soc. A 371, 20120459 (2013).

[14] L. Mei, G. Chen, L. Xu, X. Zhang, B. S. C. Gu, and A. Wang, Opt. Lett. 39, 3235 (2014).
[15] X. Yao and L. Maleki, J. Opt. Soc. Am. B 13, 1725 (1996).

[16] X. Yao and L. Maleki, IEEE J. Quant. Electron. 32, 1141 (1996).

[17] Y. Chembo, K. Volyanskiy, L. Larger, E. Rubiola, and P. Colet, IEEE J. Quant. Electron. 45, 178 (2009).

[18] Y. Chembo, L. Larger, and P. Colet, IEEE J. Quant. Electron. 44, 858 (2008).

[19] Y. Chembo, L. Larger, H. Tavernier, R. Bendoula, E. Rubiola, and P. Colet, Opt. Lett. 32, 2571 (2007).

[20] Y. C. Kouomo, Ph.D. thesis, Universitat de les Illes Balears, 2006.

[21] J. Goedgebuer, P. Levy, L. Larger, C.-C. Chen, and W. Rhodes, IEEE J. Quant. Electron. 38, 1178 (2002).

[22] N. Gastaud, S. Poinsot, L. Larger, J.-M. Merolla, M. Hanna, J. Goedgebuer, and F. Malassenet, Electron. Lett. 40, 898 (2004).

[23] K. Ikeda, H. Daido, and O. Akimoto, Phys. Rev. Lett. 45, 709 (1980).

[24] K. Ikeda and K. Matsumoto, Physica D 29, 223 (1987).

[25] T. Aida and P. Davis, IEEE J. Quant. Electron. 28, 686 (1992).

[26] J. Martínez-Llinàs, P. Colet, and T. Erneux, Phys. Rev. E 89, 042908 (2014).

[27] M. Zigzag, M. Butkovski, A. Englert, W. Kinzel, and I. Kanter, Phys. Rev. E 81, 036215 (2010).

[28] M. Peil, M. Jacquot, Y.K. Chembo, L. Larger, and T. Erneux, Phys. Rev. E 79, 026208 (2009).

[29] K. Engelborghs, T. Luzyanina, and D. Roose, ACM Trans. Math. Softw. 28, 1 (2002).

[30] J. Sieber, K. Engelborghs, T. Luzyanina, G. Samaey, and D. Roose, arXiv:1406.7144 (2015).

[31] Bram Wage, Master thesis, Utrecht University (NL), 2014.

[32] K. Engelborghs, T. Luzyanina, and G. Samaey, Technical Report TW-330, Department of Computer Science, K. U. Leuven, 2001. 